\title{
Variational Aspects of Relativistic Field Theories, with Application to Perfect Fluids
}

\author{
Bernard F. Schutz and Rafael Sorkin \\ Department of Applied Mathematics and Astronomy, University College, Cardiff, Wales
}

Received August 5, 1976

By investigating perturbations of classical field theories based on variational principles we develop a variety of relations of interest in several fields, general relativity, stellar structure, fluid dynamics, and superfluid theory. The simplest and most familiar variational principles are those in which the field variations are unconstrained. Working at first in this context we introduce the Noether operator, a fully covariant generalization of the socalled canonical stress energy tensor, and prove its equivalence to the symmetric tensor $T^{\mu \nu}$. By perturbing the Noether operator's definition we establish our fundamental theorem, that any two of the following imply the third (a) the fields satisfy their field equations, (b) the fields are stationary, (c) the total energy of the fields is an extremum against all perturbations. Conversely, a field theory which violates this theorem cannot be derived from an unconstrained principle. In particular both Maxwell's equations for $F_{\mu \nu}$ and Euler's equations for the perfect fluid have stationary solutions which are not extrema of the total energy $[(a)+(b) \nRightarrow(c)]$.

General relativity is a theory which does have an unconstrained variational principle but the definition of Noether operator is more ambiguous than for other fields. We define a pseudotensorial operator which includes the Einstein and Landau-Lifschitz complexes as special cases and satisfies a certain criterion on the asymptotic behavior. Then our extremal theorem leads to a proof of the uniqueness of Minkowski space: It is the only asymptotically flat, stationary, vacuum solution to Einstein's equations having $\mathbb{R}^{4}$ global topology and a maximal spacelike hypersurface.

We next consider perfect fluid dynamics. The failure of the extremal-energy theorem elucidates why constraints have always been used in variational principles that lead to Euler's equations. We discuss their meaning and give what we consider to be the "minimally constrained" principle. A discussion of one constraint, "preservation of particle identity," from the point of view of path-integral quantum mechanics leads to the conclusion that it is inapplicable to degenerate Bose fluids, and this gives immediately the well-known irrotational flow of such fluids. Finally, we develop a restricted extremal theorem for the case of perfect fluids with self-gravitation, which has the same form as before except that certain perturbations are forbidden in (c). We show that it is a generalization of the Bardeen-Hartle-Sharp variational principle for relativistic stellar structure. It may be useful in constructing nonaxisymmetric stellar models (generalized Dedekind ellipsoids). We also give the Newtonian versions of the main results here, and we show to what extent the extremal theorems extend to fields that may not even have a variational principle. 


\section{INTRODUCTION AND SUMmary}

\section{(a) Motivation}

Euler's equation for the motion of a compressible perfect fluid,

$$
\mathbf{V}_{, t}+(\mathbf{V} \cdot \nabla) \mathbf{V}+(1 / \rho) \nabla p=0,
$$

may be regarded as the oldest of field theories. Yet in one respect it remains one of the most puzzling: It has never been possible to derive it from a completely satisfactory variational principle. This problem has attracted considerable attention in modern times $([37,39,41]$ and references quoted in the historical appendix to Schmid [33]), but some important questions remain unanswered. Inasmuch as the entire future development of a fluid can be predicted and characterized in terms of the five "macroscopic" or "Eulerian" variables $\{\rho, p, \mathbf{V}\}$, it is sensible to ask whether one can construct a variational principle in these five variables, whose Euler-Lagrange equations are (1.1). All such variational principles known work only by imposing constraints ${ }^{1}$ on the variations of the Eulerian variables.

Although the constraints have ready physical interpretations (one, for instance, conserves particles) their necessity is not completely clear. One can eliminate them only at the price of introducing Lagrange multipliers, which then become additional fields in the theory that are not directly measureable (though perhaps not without physical interest [31]). How are we to understand the apparent absence of any variational principle in the physical variables which allows their free variation ${ }^{2}$ This question is not merely of academic interest. The possibility of deriving an equation from an unconstrained variational principle confers on it, among other things, certain self-adjointness properties that are important for the study of perturbations of its stationary solutions $[12,17]$. The theory of small perturbations of stationary solutions of Euler's equation and its near relatives is of particular interest in astrophysics and plasma physics, and an understanding of the constraints is of vital importance for the full development of that theory.

In fact, the theory of small perturbations raises another, closely related puzzle. By linearizing in the perturbation one arrives at a linear equation in terms of the variables $\{\delta \rho, \delta p, \delta \mathbf{v}\}$ : the perturbed version of (1.1). Again, this equation is apparently not derivable from any variational principle in these variables, but it does follow from one whose basic variable is the (microscopic and hence apparently extraneous) "Lagrangian displacement" vector $\xi$, which is taken to generate $\delta \rho, \delta p$, and $\delta \mathbf{v}$. This variational

\footnotetext{
${ }^{1}$ The word "constraint" for us means a restriction on the freedom to vary the fields in the Lagrangian, as is discussed further in Section 2.

2 One might answer that Euler's equation is not a "fundamental" field theory, since it results from an averaging over microscopic physics, which may conceivably throw away vital information; but this answer begs the question. What aspect of the discarded information is vital, and why is it vital? Would a "microscopic" treatment necessarily have an unconstrained variational principle? In fact, electromagnetism, a "microscopic" theory, also lacks an unconstrained variational principle in the variables $\mathbf{E}$ and $\mathbf{B}$, and we show why in Section 2.
} 
principle has much in common with the "second variation" of unconstrained Lagrangians, being symmetric and quadratic in $\xi$ [23], but it has never, to our knowledge, been derived directly as the second variation of anything. (For an indirect derivation see Schutz [35].) The Hamiltonian associated with it must be closely related to the second-order change in the energy of a stationary solution [36], but again this Hamiltonian cannot be expressed in terms of $\{\delta \rho, \delta p, \delta \mathbf{v}\}$ alone. ${ }^{3}$ This is particularly puzzling, since one knows that the energy of a fluid is a function only of $\rho, p$, and $\mathbf{v}$, so in some sense one expects this also to be true of its second-order changes.

It therefore seems to us that a close examination of the nature of the constraints on fluid variational principles is appropriate. In this paper we examine only first-order changes in functionals associated with the fluid; this proves to be the key to understanding the role of the constraints. We shall postpone considering second-order changes to a subsequent paper, because we have found that the theory of the first-order changes proves to be rich and fruitful.

Because of the variety and interconnectedness of the results we obtain, we shall now give, instead of the usual preview, a short summary of the major features of each section.

\section{(b) Plan and Summary}

Section 2. We begin with an examination of variational principles without constraints, and we assemble some powerful results that apply to any such field theory. The first question that arises is how to define energy. The convenient definition from the point of view of variational principles uses the so-called canonical stress-energy tensor (defined via Noether's theorem), but this has a number of well-known drawbacks. We introduce instead the "Noether operator," which is a completely corariant generalization of the coordinate-dependent canonical tensor, but which is still "canonical" in that its definition does not involve the metric tensor. We show in a simple way that the Noether operator always gives the same total energy, or other conserved quantity, as does the so-called symmetric tensor that is derived by varying the Lagrangian with respect to the metric. (This generalizes and simplifies the proofs of Belinfante [4] Rosenfeld [29].) We then give an equally simple proof of the main result of this section. The total energy of a stationary field is an extremum against all perturbations of the field variables if and only if the fields solve the field equations. It follows that any physical ficld whose energy does not have this remarkable extremal property cannot be derived from an unconstrained variational principle. It is on this basis that we understand the constraints in the fluid variational principles. This also applies to electromagnetism, and gives us a reason for the importance of the (locally) unmeasureable vector potential.

Section 3. The application of these results to include the metric as a dynamical field involves defining the energy of the gravitational field in general relativity. As is

\footnotetext{
${ }^{3}$ One can in fact show that this Hamiltonian is not invariant under the group of transformations of $\xi$ and $\xi_{. t}$ that leaves $\{\delta \rho, \delta p, \delta \mathbf{v}\}$ unchanged: this may be called the "particle permutation group" and is important in Section 4 below.
} 
well known, there is no locally unique way to do this, but we give an energymomentum pseudotensor which includes the Einstein and Landau-Lifshitz pseudotensors as special cases, and which is constructed to satisfy a physically desirable condition that it be as insensitive as possible to the asymptotic behavior of the gravitational field. It then follows, since vacuum general relativity possesses an unconstrained variational principle, that a stationary, asymptotically flat, vacuum solution is an extremum of the integral mass. Since the only extremum (in a wide class) is flat spacetime [5], the only stationary vacuum solution (in that class) is Minkowski space. This rules out such stationary "bound states": massless geons.

Section 4. Because the energy of a fluid is not an extremum against all changes in $\rho, S$, and $\mathbf{v}$, any variational principle in these Eulerian variables must be constrained. We identify three physically independent ways of changing a stationary fluid's energy, and show that constraints of the standard variational principles are closely related to them. Our purpose here is heuristic: to understand the constraints and their unavoidability. We then give what we consider to be the "minimally constrained" variational principle for fluids, in a form specially suited for the further development in Section 6 below.

Section 5. One of the fluid constraints can be interpreted as demanding distinguishability of the fluid's particles. Such a condition is inappropriate for a degenerate Bose fluid, but by using path-integral ideas we show that merely dropping this constraint gives a variational principle which correctly describes superfluid flow.

Section 6. Extremal theorems analogous to those of Section 2 are proved for the perfect fluid, taking into account the constraints. This yields explicit formulas for the way in which the total energy does change for those perturbations against which it is not an extremum, and also gives the result that a stationary metric and fluid are a solution of Einstein's equations if and only if their total energy is an extremum against a certain wide class of perturbations. This generalizes the Bardeen [2] variational principle to arbitrary stationary configurations (i.e., not necessarily axially symmetric). These results are, in fact, valid in a certain form for any fields which may be the source of the metric in general relativity, regardless of whether or not they are derivable from a variational principle of any sort.

Appendix. We examine more closely the relations between the perfect fluid's "minimally constrained" variational principle of Section 4 and certain unconstrained variational principles in unphysical variables.

\section{(c) Notation and Conventions}

Throughout the paper we keep to a relativistic point of view, except in certain places where the physical discussion is most easily phrased in Galilean terms. In Section 6, however, we also give the Newtonian versions of our general-relativistic theorems, because these are of interest in astrophysics. Our conventions follow Misner et al. [24]: signature +2 , Greek indices running from 0 to 3 , Latin from 1 to 3 . We shall follow the usual convention of using Gothic letters to denote tensor densities. 


\section{Field Theories Derived from Unconstrained Lagrangians}

In order to show that constraints are necessary in variational principles for perfect fluids, and to develop the techniques we will use to derive the extremal results for perfect fluids, we must first study principles without constraints. The word "constraint" has several usages in field theories derivable from Lagrangians. In theories with gauge groups it is often applied to the nondynamical field equations (for example, Coulomb's equation in electromagnetism), since they constrain the freedom to set initial data. "Constraint" has also been applied by Anderson and Bergman [1] to those equations which give algebraic relations among the momenta of degenerate field theories (of which gauge theories are a class). Neither of these meanings is intended here. since both above types of equations can usually be derived from Lagrangians by unconstrained variations. Here we are interested in constraints on the variations of the Lagrangian. Some such constraints, sometimes called integrable or holonomic, can be eliminated by adding the constraint equation times a Lagrange multiplier to the Lagrangian and varying everything, including the multiplier, freely. This now principle is unconstrained in our sense (but at the cost of introducing extra variables, which may not be physically measurable). There are other principles in which the constraints cannot be removed so easily; of these there is at present only a meager understanding. Each constrained Lagrangian must be studied separately, as we do for the perfect fluid later. The unconstrained principles, on the other hand, can be discussed in complete generality.

\section{(a) Definition of the Canonical Conserved Quantities}

Consider an action

$$
S=\int_{x} \mathscr{x}[Q] d^{4} x
$$

defined as the integral over the spacetime region $\mathfrak{X}^{*}$ of a Lagrangian $\mathbb{Q}$ which is a functional of field variables $Q . \mathbb{Q}$ may be only the Lagrangian for matter ${ }^{4}$ or it may include the (general relativistic) terms needed to bring in the metric as a dynamical field. In all cases actually arising $\mathfrak{L}=\mathfrak{L}\left(Q, \partial_{\mu} Q, \partial_{\mu \nu} Q\right)$ will depend at worst on second derivatives of the field variables, but we leave it in general form since writing out precise dependences on $\hat{\partial}_{\mu} Q$ and $\hat{c}_{\mu \nu} Q$ would only complicate things. Notice that $Q$ comprises all the variables on which $\mathscr{E}$ depends, including, if it occurs, the metric $g_{\mu v}$. We will write $q$ for the variables exclusive of $g_{\mu \nu}$, which we assume to be a collection of tensors and tensor densities. Thus, we exclude spinors entirely from our discussion. (See [40] for spinors.) We treat $\mathbb{L}$ as a density.

Let the action undergo a deformation variation $\delta_{\xi}$ in which the variation "in place" of the field variables is (minus) the Lie derivatives

$$
\delta_{\xi} Q=-\underset{\xi}{\mathfrak{f}} Q \text {. }
$$

\footnotetext{
"The term "matter" can refer to any field but the metric itself.

${ }^{5}$ The Lie derivative of a tensor with respect to $\xi$ is simply the coordinate-free generalization of the partial derivative along $\xi$. It is that tensor whose components, in a coordinate systems in which $\xi^{\mu}=$ const., equal the derivatives $\xi^{\mu} \partial_{\mu}$ of the original tensor's components. See [15] or [24].
} 
and at the same time the boundary, $\partial \mathfrak{X}$, of the region of integration is dragged by the vector field $\xi$. If we consider first a $\xi$ vanishing in the neighborhood of $\partial \mathfrak{X}$, then $\delta_{\xi} Q$ will also vanish near $\partial \mathfrak{X}$, so that (according to the definition of "variational derivative") the variation of the action will be

$$
\delta S=\int_{\mathfrak{X}}(\delta \mathfrak{Q} / \delta Q) \delta_{\xi} Q d^{4} x
$$

Here $\delta \mathfrak{L} / \delta Q$, the variational derivative of $\mathfrak{L}$, can be made explicit as

$$
\frac{\delta \mathfrak{L}}{\delta \mathscr{Q}}-\frac{\partial \mathfrak{Q}}{\partial \boldsymbol{Q}}-\partial_{\mu} \frac{\partial \mathfrak{Q}}{\partial\left(\partial_{\mu} Q\right)}+\partial_{\mu \nu} \frac{\partial \mathfrak{Q}}{\partial\left(\partial_{\mu \nu} Q\right)} \cdots
$$

(Note that in (2.3) there is implied a contraction of indices and a sum over the distinct fields involved in $Q$.)

If we now drop the restriction on $\xi$ there will appear in (2.3) a second term associated with the boundary of $\mathfrak{X}$

$$
\delta_{\xi} S=\int_{\mathfrak{X}}(\delta \mathfrak{I} / \delta Q) \oint_{\xi} Q d^{4} x+\oint_{\partial \mathfrak{X}} \stackrel{\mathfrak{T}}{N}^{\mu}{ }^{\nu} \cdot \xi^{\nu} d \sigma_{\mu},
$$

where $d \sigma_{\mu}$ is the outwardly directed surface element (not including the "Jacobian" $|g|^{1 / 2}$ since $\mathfrak{T}_{N}{ }_{\nu}$ is already a density). We call ${\underset{N}{T}}_{{ }^{\prime}}{ }_{\nu}$, which, as indicated by the dot in Eq. (2.4), is in general a differential operator on $\xi^{\nu}$, a Noether operator associated to $\mathfrak{L}$. It is not quite unique since adding to ${\underset{N}{N}}^{\mu}{ }_{\nu} \cdot \xi^{\nu}$ any term of the form

$$
\partial_{\alpha}\left(\mathfrak{F}_{\nu}^{\mu \alpha} \cdot \xi^{\nu}\right)
$$

with

$$
\mathfrak{F}^{\mu \alpha}{ }_{\nu}=-\mathfrak{F}^{\alpha \mu}{ }_{\nu}
$$

leaves unchanged the surface integral in (2.4).

Let us illustrate these definitions when, as is usual, $\mathfrak{L}=\mathfrak{I}\left(Q, \partial_{\mu} Q\right)$. Then

$$
\delta S=\int_{\mathfrak{X}} \delta \mathfrak{Q}+\oint_{\partial \mathfrak{X}} \mathfrak{Q} \xi^{\mu} d \sigma_{\mu} .
$$

But

$$
\begin{aligned}
\delta \mathfrak{L} & =\frac{\partial \mathscr{Q}}{\partial Q} \delta Q+\frac{\partial \mathfrak{Q}}{\partial\left(\partial_{\mu} Q\right)} \delta \partial_{\mu} Q \\
& =\frac{\delta \mathscr{Q}}{\delta Q} \delta Q+\partial_{\mu}\left(\frac{\partial \mathbb{Q}}{\partial\left(\partial_{\mu} Q\right)} \delta Q\right)
\end{aligned}
$$

whence

$$
\begin{aligned}
\mathfrak{T}_{N}{ }_{\nu} \cdot \xi^{v} & =\xi^{\mu} \mathfrak{Q}+\frac{\partial \mathfrak{Q}}{\partial\left(\partial_{\mu} Q\right)}{ }_{\xi} Q \\
& =\xi^{\mu} \boldsymbol{Q}-\frac{\partial \mathfrak{Q}}{\partial\left(\partial_{\mu} Q\right)} \underset{\xi}{£} Q .
\end{aligned}
$$


In the special case where $\xi$ is a constant vector (a coordinate dependent notion!)

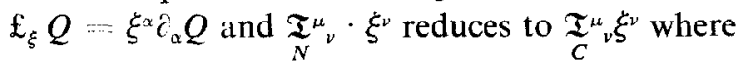

$$
{\underset{C}{\boldsymbol{T}^{\prime}}}_{\nu}=\mathbf{Q} \delta^{\mu}{ }_{\nu}-\left(\partial \boldsymbol{Q} / c\left(\partial_{\mu} Q\right)\right) \dot{c}_{\nu} Q
$$

is the familiar "canonical stress-energy tensor." We can extend this definition of $\underset{C}{T^{\mu}}{ }^{\mu}$ to higher-order Lagrangians by maintaining the relation ${ }^{6}$ :

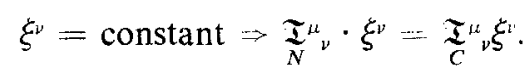

It is clear from the manner in which it was derived that (provided that $\mathbb{Q}$ is a scalar density) $\underset{N}{\mathfrak{T}^{\mu}}{ }_{\nu}$ is a tensor-density operator (i.e., $\underset{N}{\mathfrak{T}^{\mu}}{ }_{\nu} \cdot \xi^{v}$ is a vector density for any differentiable vector field $\xi^{\nu}$ ). On the other hand, the "canonical tensor" is only a pseudotensor, since it transforms as a tensor only under the restricted group of coordinate transformations that leave $\xi^{v}$ with constant components. The familiar complaints against the canonical tensor, that it is not a true tensor, and that (because it is not always symmetric) it is unsuitable for defining angular momentum, do not apply to the Noether operator, which can be used to define a "canonical" expression for any conserved quantity, as we shall show.

Using the definition (2.8) and the basic relation (2.4) it is easy to understand why ${\underset{C}{C}}^{\mathfrak{I}^{n}}{ }_{\nu}$ is conserved. Suppose that $\mathfrak{L}$ is a scalar density, or at least behaves like one under constant translations $\xi^{\mu}$, and that the equations of motion, $\delta \mathfrak{L} / \delta Q=0$, hold. Then setting $\xi^{\mu}=\xi_{0}^{\mu}=$ const in (2.4), both the L.H.S. and the first term on the R.H.S. vanish. Choosing $\mathfrak{X}$ to be bounded by two spacelike hypersurfaces $\mathscr{H}_{1}, \mathscr{H}_{2}$ and a "cylindrical" surface, $S$, at spatial infinity, we obtain

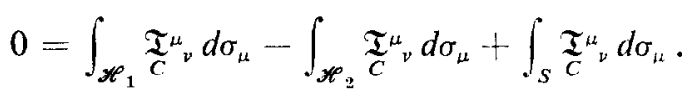

If $\widetilde{T}_{C}{ }^{\mu}$ falls off rapidly enough at spatial infinity then (2.9) just expresses the conservation of the canonical energy-momentum vector. More generally we see that

$$
P[\xi, \mathscr{H}] \equiv \int_{\mathscr{H}}{\frac{\mathfrak{T}^{\mu}}{N}}_{\nu} \cdot \xi^{v} d \sigma_{\mu}
$$

will be conserved (given the equations of motion for $\mathscr{L}$ ) whenever the action is invariant under $\xi$ and no trouble occurs at spatial infinity.

Finally we emphasize that according to (2.5) there can be many Noether operators for a given Lagrangian. The results of this paragraph will apply for any choice of $\mathfrak{T}_{N}{ }^{\mu}{ }_{\nu}$. In Section 3 we settle on a particular choice for the gravitational Noether operator but for that of matter the defining Eq. (2.4) is all we will ever need.

'A change (2.5) in the Noether operator will add to the "canonical tensor" the divergence $\partial_{\alpha} \widetilde{\mho}^{\mu_{x}}{ }_{\nu}$. 
(b) Equivalence of the Canonical and Covariant Stress-Energy Tensors for Matter

Both the Noether operator for matter, $\mathfrak{T}_{N}{ }^{\mu}$, and the "symmetric" stress tensor (density)

$$
\mathfrak{T}^{\mu \nu}=2\left(\delta \mathfrak{E}_{m} / \delta g_{\mu \nu}\right)
$$

are defined in terms of variations of the matter Lagrangian $\mathfrak{I}_{m}$. It is often asserted that $\mathfrak{T}_{\mathcal{C}}{ }_{\nu}$ and $\mathfrak{I}^{\mu \nu}$ give the same total energy-momentum. Using our definitions we can establish in a very general context the equivalence ( $\mathscr{H}$ being a hypersurface which is asymptotically flat and spacelikc ${ }^{8}$ )

$$
\int_{\mathscr{H}} \mathfrak{\mathfrak { T }}_{N}{ }_{\nu} \cdot \xi^{\nu} d \sigma_{\mu}=\int_{\mathscr{H}} \mathfrak{T}^{\mu \nu} \xi_{\nu} d \sigma_{\mu} .
$$

With appropriate choices of $\xi$ this produces equality not only for energy and momentum but for angular-momentum as well. In this subsection $\mathfrak{E}$ will mean $\mathfrak{L}_{\text {matter }}$ only, while $Q=\left\{q, g_{\mu v}\right\}$ are, as always, all the variables on which $\mathfrak{E}$ depends.

THEOREM 2.1. Assume that $\mathfrak{L}=\mathfrak{L}_{\text {matter }}$ is a scalar density. If the equations of motion for matter hold

$$
\delta \mathfrak{S} / \delta q=0
$$

then for any vector field $\xi$ and any spacetime region $\mathfrak{X}$,

$$
\int_{\partial \mathfrak{X}} \mathfrak{T}_{N}{ }_{\nu} \cdot \xi^{\nu} d \sigma_{\mu}=\int_{\partial \mathfrak{X}} \mathfrak{T}^{\mu \nu} \xi_{\nu} d \sigma_{\mu} .
$$

Proof. Breaking $Q$ into its gravitational and matter parts, and using (2.11) and (2.12), we obtain

$$
\begin{aligned}
(\delta \mathfrak{Q} / \delta Q) \delta_{\xi} Q & =\frac{1}{2} \mathfrak{T}^{\mu \nu} \delta_{\xi} g_{\mu \nu}+(\delta \mathfrak{E} / \delta q) \oint_{\xi} q \\
& =-\mathfrak{T}^{\mu \nu} \nabla_{\mu} \xi_{\nu}-(\delta \mathfrak{E} / \delta q) \underset{\xi}{\mathfrak{\xi}} q .
\end{aligned}
$$

Also, $\underset{\xi}{\delta} S=0$ since $\mathfrak{E}$ is a scalar (density), so that (2.4) becomes, after an integration by parts

$$
\oint_{\partial \mathfrak{X}}\left(\mathfrak{T}_{N}{ }_{\nu} \cdot \xi^{\nu}-\mathfrak{T}^{\mu \nu} \xi_{\nu}\right) d \sigma_{\mu}=\int_{\mathfrak{X}}\left[\frac{\delta \mathfrak{L}}{\delta q} \underset{\xi}{\mathfrak{\xi}} q-\left(\nabla_{\mu} \mathfrak{T}^{\mu \nu}\right) \xi_{\nu}\right] d^{4} x
$$

7o our knowledge the nearest thing to a published proof is represented by Eqs. (20) and (11) of Rosenfeld [29] from which one could derive, in the case where $\mathfrak{L}$ is of the form $\mathfrak{E}\left(Q, \partial_{\mu} Q\right)$, that $\mathfrak{T}_{C}^{\mu}{ }_{\nu}$ and $\mathfrak{T}^{\mu \nu}$ differ by a divergence.

${ }^{8}$ We can call such a hypersurface "asymptotically regular." Later on, in dealing with gravity we will have to strengthen the regularity conditions considerably. 
Since $\delta \mathfrak{I} / \delta q=0$ by hypothesis, and since $\xi^{\nu}$ is arbitrary everywhere on $\mathfrak{X}$, the two sides of (2.14) must vanish separately. This means

$$
\nabla_{\mu} \mathfrak{T}^{\mu \nu}=\mathbf{0}
$$

(the usual deduction from $\delta \mathfrak{L} / \delta q=0$ ) and also establishes the theorem.

It remains to argue from (2.13) to (2.12). For simplicity assume that the matter is restricted to a compact spatial region. (This is just a convenience except for zero restmass fields, for which one must examine convergence at spatial infinity in somewhat more detail. We look at this briefly in Section 3.) In (2.13) let $\mathfrak{X}$ be the region between two asymptotically regular hypersurfaces $\mathscr{H}$ and $\mathscr{H}^{\prime}$ and let $\mathscr{N}$ be a compact set which excludes $\mathscr{H}^{\prime}$ but includes (in its interior) the intersection of the matter with $\mathscr{H}$ (Fig. 1). By choosing $\xi=0$ outside $\mathscr{N}$ but otherwise arbitrarily, (2.13) becomes (2.12) since the latter involves $\xi$ only within $\mathscr{N}$.

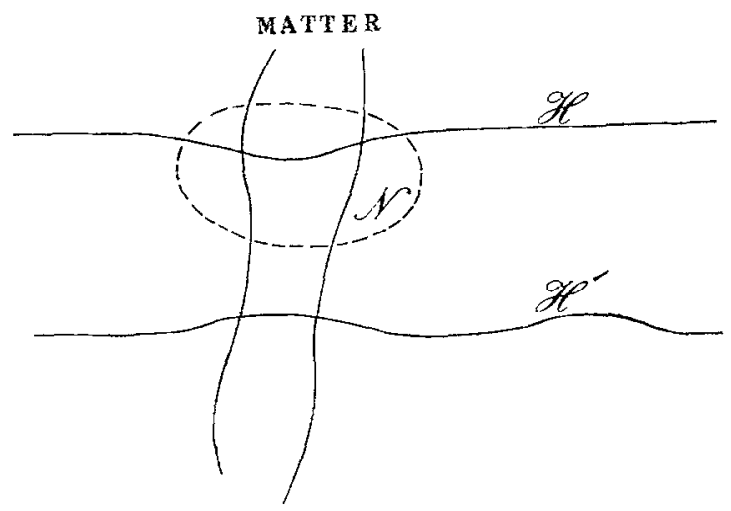

FIG. 1. Spacetime diagram of the region including the spacelike hypersurfaces $\mathscr{H}^{\mathscr{t}}$ and $\mathscr{H}^{\prime}$. The matter between the hypersurfaces is confined to a region of compact support, its boundary being indicated by the solid timelike lines. The vector field $\xi$ vanishes outside $f$ in the discussion at the end of Section $2 b$, while in the proof of Theorem 2.2 the perturbation $\delta Q$ vanishes outside $r$.

\section{(c) Extremum Principle for the Total Energy}

Consider a point-mass moving in a potential well. In this familiar problem of classical mechanics the only static solutions have the particle resting at a position of zero slope. And in such a situation not only the potential, but also the total energy is an extremum; for since the particle is at rest, any variation of its velocity affects the kinetic energy only to second order. In other words, a configuration which is (a) stationary, and (b) a solution of the equation of motion must also be (c) an extremim of the total energy.

This relation is part of a more general one: of the three conditions (a), (b), (c), any pair implies the third. The reader can easily convince herself or himself of this by thinking through the other two implications. 
In the present section we generalize this simple relation to any field theory based on an unconstrained variational principle. On this generalization will rest our explanation of why there is no perfect-fluid action principle based on unconstrained variation of Eulerian variables.

In contrast to the notation of the last subsection let $\mathfrak{I}$ now be the total Lagrangian for some system of fields (including therefore $g_{\mu \nu}$ ) and suppose that $\xi^{\mu}$ is a deformation which leaves the action unchanged. (If $\mathfrak{L}$ is a true scalar density then any $\xi$ will do, but one might want to use for gravity the Einstein "first-order" Lagrangian, which is only an affine density. Then, if gravity were involved, $\xi^{\mu}$ would have to be linear in the coordinates.)

According to (2.2) and (2.4) we have for such a $\xi$, and for any region $\mathfrak{X}$,

$$
\int_{\mathfrak{X}} \mathfrak{E} \underset{\xi}{£} Q d^{4} x=\oint_{\partial \mathfrak{X}}{\underset{\mathfrak{T}}{N}}_{\nu}^{\mu} \cdot \xi^{\nu} d \sigma_{\mu}
$$

where we have put for short

$$
\mathfrak{E} \equiv \delta \mathfrak{L} / \delta Q
$$

the vanishing of which constitutes the equations of motion or "Euler-Lagrange" equations.

Now vary $Q$ arbitrarily without changing $\xi$ or $\mathfrak{x}$

$$
\int_{\mathfrak{X}} \delta \mathfrak{E} \underset{\xi}{\mathfrak{E}} Q d^{4} x+\int_{\mathfrak{X}} \mathfrak{E} \underset{\xi}{\mathfrak{E}} \delta Q d^{4} x=\oint_{\partial \mathfrak{X}} \delta \underset{N}{\mathfrak{T}}{ }_{\nu} \cdot \xi^{\nu} d \sigma_{\mu} .
$$

Using this identity we will prove

THEOREM 2.2. Let $\mathfrak{L}$ be the Lagrangian for an unconstrained variational principle. Let $\xi^{\mu}$ be a vector field for which $\delta_{\xi} S=0$ and define for any hypersurface $\mathscr{H}$,

$$
P[\xi, \mathscr{H}] \equiv \int_{\mathscr{H}}{\underset{\mathfrak{T}}{\mu}}^{\mu} \cdot \xi^{\nu} d \sigma_{\mu}
$$

as the total associated " $\xi$-momentum." Then of the following conditions any two imply the third ${ }^{9}$ :

(a) $Q$ is stationary with respect to $\xi: \underset{\xi}{£} Q=0$,

(b) $Q$ is a solution: $\delta \mathscr{Q} / \delta Q=0$ wherever $\xi \neq 0$,

(c) For any asymptotically regular hypersurface $\mathscr{H}, P[\xi, \mathscr{H}]$ is an extremum against all variations $\delta Q$ of compact spacetime support.

Proof. (a) + (b) $\Rightarrow$ (c). In (2.18) the L.H.S. vanishes by hypothesis. Therefore

$$
\oint_{\partial \mathfrak{X}} \delta \mathfrak{T}_{N}^{\mu} \cdot \xi^{\nu} d \sigma_{\mu}=0 .
$$

${ }^{9}$ There is also a very mild "generality" assumption in the proof that (b) + (c) $\Rightarrow$ (a). 
Let $\delta Q$ be of compact support, but otherwise still arbitrary. Then we can, as in Fig. 1, choose $\mathfrak{X}$ as the region between $\mathscr{H}$ and a second hypersurface $\mathscr{H}^{\prime}$ lying away from the support of $\delta Q$. Equation (2.20) reduces to condition (c)

$$
\delta P[\xi, \mathscr{H}]=0 .
$$

(b) + (c) $\Rightarrow$ (a). We claim that (c) implies the vanishing of the R.H.S. of (2.18) for all regions $\mathfrak{X}$. For by choosing $\mathscr{H}^{\prime}, \mathscr{H}^{\prime \prime}$ as shown in Fig. 2 , we can arrange that the R.H.S. of (2.18) is just

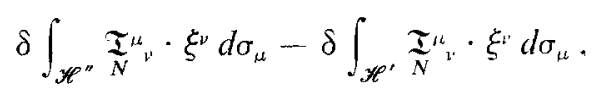

hoth of which vanish by (c). Using (b) we get (since $\mathfrak{X}$ is arbitrary)

$$
\delta \in \underset{\xi}{\mathfrak{f}} Q=0 \quad \text { for all } \delta Q .
$$

Except in very special cases $\delta \mathfrak{E}$ will be a general variation and we conclude $\underset{\xi}{\mathfrak{f}} Q=0$.

(c) + (a) $\rightarrow$ (b). Now we get from (2.18) (arguing as before for (c))

$$
\int_{\mathfrak{X}} \underset{E}{\mathfrak{E}} \delta Q=0 \quad \forall \dot{X}, \forall \delta Q
$$

Since $\mathfrak{Z}$ is arbitrary, the integrand vanishes for any $\delta Q$, and since $\underset{\xi}{£}$ preserves the generality of $\delta Q$ where $\xi \neq 0, \mathbb{E}=0$ there.

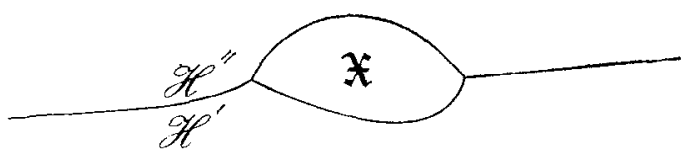

FIG. 2. The hypersurfaces $\mathscr{H}^{\prime}$ and $\mathscr{H}^{\prime \prime}$ coincide outside the region $\mathfrak{X}$. Since the only regularity conditions on $\mathscr{H}^{\prime}$ and $\mathscr{H}^{\prime \prime}$ are asymptotic (at spatial infinity), $\mathfrak{X}$ is arbitrary.

What is the meaning of $P[\xi, \mathscr{H}]$ ? When $\xi$ is a killing vector of the background metric $g_{\mu \nu}$ and $\mathfrak{T}_{N}{ }_{\nu}$ is formed from the matter Lagrangian alone then $P[\xi, \mathscr{H}]$ is just the conserved quantity associated with the symmetry generated by $\xi$. More generally, if, as in Section 3 below, $g_{\mu \nu}$ is included as a dynamical field by adding an appropriate term to $\mathbb{E}$ then the $\xi$-momentum will still have physical sense if spacetime is asymptotically fiat and $\xi$ becomes a killing vector at spatial infinity. In particular, when $\xi^{u}=$ const, $P[\xi, \mathscr{H}]$ just gives (a component of) the total four-momentum, as discussed in detail in Section 3.

(d) A Simple Application to the Electromagnetic Field

Our main concern in this paper is with the theory of the perfect fluid, which we treat in Sections 4-6. We pause here, however, to point out an interesting consequence of the theorem just proved. 
COROLLARY 2.3. There exists no unconstrained variational principle for Maxwell's equations in which the field variable is the electromagnetic tensor $F_{\mu \nu}$ (equivalently, the electric and magnetic fields, $\mathbf{E}$ and $\mathbf{B})$.

Proof. We know (from experiment) that the energy of a stationary electromagnetic field is $\int\left(E^{2}+B^{2}\right) / 8 \pi d^{3} x$. But as this is obviously not an extremum against all possible changes in $\mathbf{E}$ and $\mathbf{B}$ of compact support, there cannot be an unconstrained variational principle in $\mathbf{E}$ and $\mathbf{B}$ from which Maxwell's equations can be deduced.

There are two ways to construct a variational principle for Maxwell's equations consistent with this corollary, and both are well known. The first is to constrain the variations: one uses $F_{\mu \nu}$ but demands that $\delta F_{[\mu \nu, \nu]}=0$. The second way is to introduce the vector potential as the field variable, which permits an unconstrained variational principle in a (classically) unmeasureable potential. This gives an interesting reason for the necessity of using "unphysical" potentials in classical field theories. ${ }^{10} \mathrm{In}$ Section 4 we will prove in the same manner that no unconstrained variational principle in "physical" variables exists for the perfect fluid.

\section{On the Inclusion of the Metric as a Dynamical Field}

In this rather technical section we want to show that the long-range character of gravity does not invalidate the conclusions set forth above. In other words, the inclusion of the metric as a dynamical field will not violate the proviso that things be "OK at spatial infinity." In particular, we will be able to free the variations contemplated in Theorem 2.2 from the restriction that they be of compact support. We also clarify a subtle distinction between the total mass as we define it, and the mass which appears "at infinity" as a parameter in the Schwarzschild metric.

In the final subsection we combine the work of Section $2 \mathrm{c}$ with a result of Brill and Deser to rule out the existence of "bound states" of the gravitational field.

\section{a. A Noether Operator for Gravity}

Because gravity, like electromagnetism, is a long-range field, various surface terms (at spatial infinity) that were discarded in the above work might, in principle, cause trouble. In fact, since the neglected terms often look like $\phi \partial \phi$ where $\phi$ is the appropriate potential $\left(g_{\mu \nu}\right.$ or $A_{\mu}$ ), the danger is even greater for gravity than with electromagnetism. For one usually assumes that $A_{\mu} \rightarrow 0$ at infinity, but such an assumption about $g_{\mu \nu}$ is inconsistent with asymptotic flatness. Therefore the $g \partial g$ term falls off only like $1 / r^{2}$ where $A \partial A$ would fall off like $1 / r^{3}$. (One can therefore anticipate that any regularity conditions we verify for gravity will be true a fortiori for any other null field.)

All this has the effect that not all naturally arising choices for the gravitational Noether operator (Section 2a) are equivalent in the sense of Eq. (2.12). They yield

\footnotetext{
${ }^{10}$ In Section 5 we tentatively distinguish between "essential" and "inessential" constraints. In this terminology the electromagnetic constraint would be inessential.
} 
different values for the total mass, and especially for the total angular momentum. We do not propose to discuss this in any more detail, but only to present a choice for the gravitational ${\underset{\mathfrak{T}}{\boldsymbol{v}}}_{v}^{\mu}$ which will allow us, when dealing with the total mass, to include terms for the dynamical metric in $\mathfrak{l}$ without invalidating the results of Section 2.

As the Lagrangian for gravity we use

$$
(1 / 16 \pi)(-g)^{1 / 2} R
$$

where $R$ is the curvature scalar. Carrying out the variation as in Section 2 a gives

$$
\delta R / \delta g_{\mu \nu}=-G^{\mu \nu}
$$

(where $\delta R / \delta g_{\mu v} \ldots(1 /-g)^{1 / 2}\left(\delta\left((-g)^{1 / 2} R\right) / \delta g_{\mu v}\right)$ ) so that, according to (2.4).

$$
8 \pi \underset{\varrho}{\delta} S==\frac{T}{2} \int G^{\mu \nu} \underset{\xi}{\underset{\xi}{f}} g_{\mu \nu} d^{4} x \cdot 8 \pi \oint \frac{\mathfrak{T}_{N}^{\mu}}{\nu^{\nu}} \cdot \xi^{\nu} d \sigma_{\mu} .
$$

But since $R$ is a scalar which depends on $g_{\mu \nu}$ alone, $\underset{\xi}{\delta} S==0$ and (3.3) becomes

$$
0=\int G^{\mu \nu} \nabla_{\mu} \xi_{v} d^{4} x+8 \pi G \oint \frac{\mathfrak{T}^{\mu}}{\mathfrak{N}^{v}} \cdot \xi^{v} d \sigma_{u}
$$

An integration by parts, together with the identity $\Gamma_{\mu} G^{\mu v}-0$, then shous that, as suggested by Lorentz, ${ }^{11}$

$$
8 \pi{\underset{N}{N}}^{\mu}=-(-g)^{1 / 2} G_{1}^{\mu}
$$

would be a possible definition.

Such a choice suffers from two drawbacks. First $\underset{N^{v}}{T^{\mu}}$ would include terms linear in the second derivatives, $g_{\mu \nu, \alpha \beta}$, whereas for the next section we would like it to be a quadratic form in the first derivatives, $g_{\mu v, \alpha}$. Second and more seriously, the total mass (matter - metric) would vanish for any solution of the Einstein equations.

$$
G^{\mu \nu}=8 \pi T^{\mu \nu}
$$

as is obvious from (3.5) and (2.12) for matter. By taking advantage of the ambiguity (2.5) in $\underset{N^{\nu}}{\mathfrak{T}^{\mu}}$ we can solve both these problems by adding an appropriate (noncovariant!) divergence to (3.5).

Accordingly we define the gravitational Noether operator by

$$
8 \pi \underset{\mathbf{N}^{\nu}}{\mu} \cdot \xi^{\nu}=-(-g)^{1 / 2} G_{\nu}^{\mu} \xi^{\nu}+\frac{1}{y} C_{x}\left(h^{\mu \alpha \nu \beta}{ }_{\beta} \xi_{\nu /}(-g)^{1 / 2}\right)
$$

where

$$
h^{\mu \nu \alpha \beta}=(-g)\left(g^{\mu \alpha} g^{\nu \beta}-g^{\nu \alpha} g^{\mu \beta}\right) .
$$

${ }^{11}$ An excellent review of previous work on conservation laws is given by Trautman [45]. 
It is a straightforward if tedious matter to verify that all the second derivatives of $g_{\mu \nu}$ cancel from this expression. Furthermore, when $\xi^{\prime \prime}=$ constant it becomes the socalled Einstein pseudotensor [45] whereas when $\xi_{\nu}=$ (const) $(-g)^{1 / 2}$ it plainly reduces to the Landau-Lifschitz expression [18, Section 100]. Because the second-order terms cancel from (3.7) one sees that both the Einstein and Landau Lifshitz expressions will be quadratic in the $g_{\mu v, \alpha}$.

We should emphasize that (3.7), unlike the Noether operator for matter, is not generally covariant. By adding a different divergence to (3.5) one can make a covariant operator [16] but this is of second differential order in $g_{\mu \nu}$. The virtue of our choice is its relative insensitivity, as shown in Lemma 3.1, to the precise manner in which $\xi^{\mu}$ becomes constant, and the coordinate system becomes Galilean, at spatial infinity (the condition on the coordinate system in addition to that on $\xi$ being needed here because ${\underset{v}{v}}_{\nu}^{\mu} \cdot \xi^{\nu}$ is now only a pseudovector density). Notice also that because the coordinate dependent term in (3.7) is a divergence, it never need be evaluated, in evaluating $P[\xi, \mathscr{H}]$ say, except as $r \rightarrow \infty$. The lack of covariance is thus not as much of a drawback as it might otherwise seem.

(b) Relation of the $\xi$-Momentum to the Total Mass

Until now we have been using $\mathfrak{T}_{\nu}^{\mu}$ for the Noether operator of any Lagrangian. Henceforth we have to distinguish the gravitational from the matter terms. We adopt the convention that upper case letters,

$$
\mathfrak{T}^{\mu \nu} \quad \text { and } \quad \mathfrak{T}_{N^{\nu}}^{\mu}
$$

refer to matter alone, while $\underline{N}_{v}^{\mu}$ will be the gravitational operator defined in (3.7) and (3.8). The $\xi$-momentum of the total Noether operator is thus

$$
P[\xi, \mathscr{H}]=\int_{\mathscr{H}}\left(\boldsymbol{T}_{\boldsymbol{N}}^{\mu} \cdot \xi^{\nu}+\mathbf{N}^{\nu} \cdot \xi^{\nu}\right) d \sigma_{\mu}
$$

Suppose the field equations for matter are fulfilled and $\xi^{\mu}=$ const. Then according to (2.12) we can replace $\mathfrak{T}_{N}^{\mu}$ by $\mathfrak{T}_{\nu}^{\mu}$, while ${\underset{N}{\nu}}_{\nu}^{\mu}$ becomes the Einstein pseudotensor. Thus $P$ is what is usually identified as (the $\xi$-component of) the total four-momentum of the system. In particular if $\xi^{\mu}=\delta_{0}^{\mu}$ then $P[\xi, \mathscr{H}]$ is the total mass, $M$.

If the field equations for the metric (i.e., the Einstein equations) are fulfilled as well, $M$ will express itself in the asymptotic behaviour of $g_{\mu \nu}$; for substituting for $\mathrm{t}_{\nu}^{\mu}$ from (3.7) and using (3.6), we find

$$
P[\xi, \mathscr{H}]=(1 / 16 \pi) \int_{\mathscr{H}} \partial_{\alpha}\left(h^{\mu \alpha \nu B}{ }_{\beta} \xi_{\nu} /(-g)^{1 / 2}\right) .
$$

By (3.8) the expression in parenthesis is skew in $\mu$ and $\alpha$ and therefore converts into an integral on $\partial \mathscr{H}$, i.e., at spatial infinity. It is easy to check that when (1) $g_{\mu \nu}$ is asymptotically Schwarzschild (in rectangular coordinates), (2) $\xi^{\mu}$ approaches $\delta_{0}^{\mu}$, and (3) $\mathscr{H}$ is the surface $t=0$, then this integral is just $M$.

On the other hand, the expression (3.9) makes sense whether or not any field equa- 
tions are satisfied. Not only does this enable us to define $M$ independently of any initial-value constraints, but we claim that, expressed in this way, $M$ is, in the absence of gravitational radiation at spatial infinity, also independent of the asymptotic behaviour of the metric. We put this more precisely as

Lemma 3.1. Let $g_{u v}=\eta_{\mu \nu}+h_{u v}$, where $\eta_{\mu \nu}$ is the Minkowski metric in Cartesian coordinates. and suppose that, as $r \rightarrow \infty$.

$$
\begin{aligned}
\text { (i) } h_{u v} & \text { is } O\left(r^{-(1+\epsilon \epsilon / 2)}\right) \text {, } \\
\text { (ii) } c_{a} h_{\beta \gamma} & \text { is } O\left(r^{-((3+\epsilon) / 2)}\right) \text {, }
\end{aligned}
$$

where $\epsilon$ is some positive constant. Let $\hat{g}_{\mu \nu}=\eta_{\mu \nu}+\hat{h}_{\mu \nu}$ be any other asymptotic hehavior honoring (i) and (ii). Let I be any integral of the form indicated symbolically $b y$.

$$
I=\int \operatorname{ch} c h d^{3} x \text {. }
$$

Then the asymptotic behavior of $g_{\mu \nu}$ can be changed to that of $\hat{g}_{\mu \nu}$ with an arbitrarily small change of $I$. In particular I itself converges as $r \rightarrow \infty$.

Remark. This is an appropriate place to make more precise our notion of asymptotic regularity. An asymptotically regular pair $(\mathscr{H}, \xi)$ satisfies the following. (a) $\mathscr{H}$ is a three-manifold without boundary and with a single asymptotic region in which it becomes flat and spacelike. (b) In some spacetime neighborhood of its asymptotic region there exist coordinates such that (i) and (ii) of the lemma hold. (c) Where applicable, the vector field $\xi^{\mu}$ becomes constant as $r \rightarrow \infty$. These requirements suffice for treating four-momentum; still stronger ones are needed for angular momentum. We hope to discuss angular momentum more fully in a future paper. However, all theorems of the present paper in which we do not make the restriction to translational killing vectors explicit will apply as well to angular momentum, provided one makes the correct choice of Noether operator and the correct definition of "asymptotic regularity."

Proof. Let $R$ be a fixed radius, and "patch" $g$ to $g$ in the region $R<r \cdots 2 R$ by replacing $g$ with the mixture

$$
t g_{\mu \nu}+(1-t) \hat{g}_{\mu \nu}
$$

where $0 \leqslant t \leqslant 1$ and $t=t(r)=1$ for $r<R, 0$ for $r>2 R$. (For definiteness we use $t=f(r / R)$ where $f(x)$ is any smooth function which vanishes for $x>2$ and $\ldots 1$ for $x<1$.) Then I acquires an addition consisting of three types of terms, which we can indicate symbolically by

$$
\begin{aligned}
& I_{0}=\int d t \partial h h, \\
& I_{1}=\int t \partial h \partial h, \\
& I_{2}=\int t t \partial h d h .
\end{aligned}
$$

We claim that all three vanish as $R \rightarrow \infty$. 
In fact we clearly can arrange that

$$
\left|\partial_{k} t\right|=\left|\left(x_{k} / r\right)(d t / d r)\right| \leqslant 2 / R .
$$

Then by (i), (ii) there is some constant $C$ such that

$$
\begin{aligned}
\left|I_{0}\right| & \leqslant C \int_{R}^{2 R}|\partial t||\partial t| \frac{r^{2} d r}{r^{1+\epsilon}} \\
& \leqslant \frac{4 C}{R^{2}} \int_{R}^{2 R} r^{1-\epsilon} d r \\
& \leqslant \frac{16 C}{2-\epsilon} R^{-\epsilon}, \\
\left|I_{1}\right| & \leqslant C \int_{R}^{2 R} 1 \cdot \frac{2}{R} r^{-2-\epsilon} r^{2} d r \\
& \leqslant \frac{4 C}{1-\epsilon} R^{-\epsilon}, \\
\left|I_{2}\right| & \leqslant C \int_{R}^{\infty} 1 \frac{r^{2}}{r^{-1}} \\
& \leqslant C \int_{R}^{\infty} \frac{d r}{r^{1+\epsilon}} \\
& \leqslant \frac{C}{\epsilon} R^{-\epsilon} .
\end{aligned}
$$

Since $\epsilon$ and $C$ are constants, $I \rightarrow 0$ as $R \rightarrow \infty$. $\square$

COROLLAKY 3.2. Any solution of the field equations without gravitational radiation at spatial infinity can be given the asymptotic behavior of any other sich solution without changing the gravitational energy, $P[\xi, \mathscr{H}]$, by more than an arbitrarily small amount.

Proof. According to (3.9) the gravitational energy is

$$
\int_{\mathscr{H}} \mathfrak{t}^{\mu}{ }^{\nu} \cdot \xi^{\nu} d \sigma_{\mu}
$$

where $\mathscr{H}$ is asymptotically flat and spacelike and $\xi^{v} \rightarrow \delta_{0}^{\nu}$ in asymptotically galilean coordinates. With our choice of ${\underset{N}{\nu}}_{\nu}^{\mu}$ this is of the form (3.11). In particular if $\xi^{\nu}$ is a timelike killing vector and $\xi^{v}=\delta_{0}^{\nu}$ then the term in $\partial_{\alpha} \xi_{v}=\partial_{\alpha} g_{v 0}$ in formula (3.7) is of this form.

The assumption about gravitational radiation means (see e.g. [24], Eq. (19.3)) that (i) and (ii) of the lemma will hold with $\epsilon=1$, if the coordinates are chosen appropriately.

COROLlaRY 3.3. In Theorem 2.2 suppose that $\mathscr{H}$ and $\xi$ are asymptotically regular. Rather than the restriction to compact support in (c) of that theorem one needs only that any variation preserve the conditions (i) and (ii) of Lemma 3.1. 
Proof. Only the implication (a) + (b) $\Rightarrow$ (c) has been strengthened. But by the proof of the first corollary, any variation of the more general sort is (to within an arbitrarily small difference) equivalent in its effect on $M$ to one of compact support.

This corrolary is important to us because any variation which relates two solutions will in general change the asymptotic behavior of the metric. Therefore Theorem 2.2 in its original form would not apply to most systems of astrophysical interest.

Because of the importance of "asymptotic patching" (Lemma 3.1), we wish to give a physical argument which suggests that any reasonable choice of $\mathfrak{N}^{u}$ must be sufficiently insensitive to the asymptotic behavior of the metric to permit such patching. Consider two solutions to Einstein's equations. The first is arbitrary except that it be asymptotically flat, and free of matter and gravitational radiation outside some radius $R$. Then its total mass is the integral

$$
M=\int_{\mathscr{H}}\left(\mathrm{t}^{\mu}{ }^{\nu} \cdot \xi^{v}+\mathfrak{I}^{\mu} \xi^{\nu}\right) d \sigma_{\mu},
$$

and this mass is reflected in the asymptotic behavior of the metric outside $R$. The second system is identical to the first except that a small amount of matter with negligible self-gravitation has been added at some radius $R^{\prime} \gg R$ (so that its "gravitational potential energy" in the field of the rest of the system is also negligible). Then the matter tensor is $\mathfrak{Z}_{\nu}^{\prime \mu}$, and because there is no self-gravitation a reasonable definition of the total mass should give an extra mass in the second system of

$$
\delta M=\int_{\mathscr{H}}\left(\mathfrak{I}_{\nu}^{\prime \mu} \xi^{\nu}-\mathfrak{I}^{\mu} \xi^{\nu}\right) d \sigma_{\mu} .
$$

If we call the gravitational Noether operator for the second system $t_{v}^{\prime \mu}$, then we have from (3.15) and (3.9)

$$
0=\int\left(\mathfrak{t}_{\nu}^{\prime \mu} \cdot \xi^{v}-\mathfrak{t}_{N}^{\mu} \cdot \xi^{\nu}\right) d \sigma_{u} .
$$

On the other hand, the metric tensor of the second system has a different asymptotic behavior from that of the first, changing in some smooth way between $R$ and $R^{\prime}$ in order to reflect the added mass. Then Eq. (3.16) expresses the requirement that the Noether operator be insensitive to this change. (Obviously the change must be suitably smooth. Gravitational waves between $R$ and $R^{\prime}$ should change the gravitational part of the total mass.) This insensitivity can be interpreted, as we have just shown, as a requirement that the definition of the total mass in (3.14) be "normalized" to the definition used for nongravitational fields.

\section{(c) Application of Theorem 2.2 to Empty Space}

THEOREM 3.4. Any stationary ${ }^{12}$ solution to the Einstein vacuum field equations is an extremum of the integral mass.

${ }^{12}$ Our term "stationary" in what Carter [8] refers to as" "pseudostationary": the Killing vector is timelike at infinity but may be spacelike elsewhere. 
Proof. By assumption there is an asymptotically timelike killing vector $\xi^{\mu}$, which rules out gravitational radiation. Then applying Theorem 2.2 as extended by Corollary 3.3 shows that $g_{\mu \nu}$ extremizes $M=P[\xi, \mathscr{H}]$ compared, in particular, to any nearby solution $g_{\mu \nu}^{\prime}$.

COROLlaRY 3.5. The only asymptotically flat, stationary vacuum solution having a maximal spacelike hypersurface with $\mathbb{R}^{3}$ topology is Minkowski spacetime.

Proof. Brill and Deser [5] have shown that the only extremum of the total mass which is asymptotically flat and entirely vacuum, and which has the assumed hypersurface is Minkowski spacetime. Since our definition of the mass agrees with the one they used when $g_{\mu \nu}$ is a solution, Theorem 3.4 applies.

We know of two other ways of obtaining a similar result about the uniqueness of Minkowski space. The first consists of combining the proof of Serini [38], discussed in Pauli [27]) that Minkowski space is the only static vacuum spacetime having $\mathbb{R}^{4}$ topology, with the Lichnerowicz theorem $[20,21]$ that a strictly stationary vacuum spacetime with $\mathbb{R}^{4}$ topology is static. This is almost identical to our Corollary 3.5 , except that our condition that the spacetime admit a maximal spacclike slice is replaced by the condition that it be strictly stationary (timelike Killing vector everywhere). It may be that these two conditions are closely related. It may prove possible to show that all strictly stationary spacetimes do, in fact, have a maximal spacelike hypersurface. ${ }^{13}$ Conversely, if it happens that spacetimes having ergoregions do not have maximal spacelike hypersurfaces, then it may still be possible to construct stationary geons.

The second method of proof uses the recent theorem of $\bar{O}$ Murchadha [25] that every stationary spacetime having a spacelike maximal slice with $\mathbb{R}^{3}$ topology and whose source obeys the strong energy condition has positive mass. In the vacuum case a stationary metric must have zero mass [18] and one can use this to show that the metric is therefore static. This in turn, through the field equations, implies that the whole spacetime is flat. The interesting feature of this approach is that it is a variant of the Lichnerowicz theorem, showing that stationarity plus maximality implies staticity. This is further evidence of the close relationship of maximality and strict stationarity.

\section{Variational Principles for Perfect Fluids}

\section{(a) Absence of an Unconstrained Variational Principle}

A perfect fluid is defined to be a fluid with isotropic pressure, no viscosity, and (in relativity) no heat conduction. ${ }^{14}$ At each event it is characterized by its four-velocity $U^{\alpha}$ and the following scalars, all measured in a local Lorentz frame moving with the

\footnotetext{
${ }^{13}$ Cantor et al. [7] have shown that possession of a maximal hypersurface is a property of a spacetime stable against small perturbations.

${ }^{14}$ For a thorough discussion of fluids in relativity the reader may consult Thorne [43].
} 
fluid at that event: the density of total mass-energy, $\rho$; the number density of conserved particles, $n$; the pressure, $p$; the specific entropy, $S$; the temperature, $T$; and the relativistic enthalpy, $\mu \equiv(\rho+p) / n$. Only two of these scalars are independent [11]: Once an equation of state, such as $\rho=\rho(n, S)$, is given, all other variables are derivable from it by the familiar rules of thermodynamics. The fluid therefore has five independent macroscopic variables: two parameters of the equation of state and three components of the four-velocity. Four dynamical equations among them are

$$
\nabla_{\alpha} T_{\beta}^{\alpha}=0
$$

where

$$
T_{\beta}^{\alpha}=\mu n U^{\alpha} U_{\beta}+p \delta^{\alpha}{ }_{\beta}
$$

A fifth equation is required to make the system well determined, and it can be either conservation of particles or of entropy

$$
\nabla_{\alpha}\left(n U^{\alpha}\right)=0 \quad \text { or } \quad U^{a} \partial_{\mathrm{n}} S=\cdots 0
$$

Either one of (4.3) plus (4.1) implies the other of (4.3) [see Eq. (4.16)].

The Newtonian limit of the spatial part of $(4.1)$ is

$$
\partial \mathbf{v} / \partial t+(\mathbf{v} \cdot \nabla) \mathbf{v}+\nabla \Phi+\rho^{-1} \nabla p=0
$$

where $\mathbf{v}$ is the three-velocity, and $\Phi$ the Newtonian gravitational field. This is the familiar Euler equation.

Equations (4.1) and (4.3) form a self-consistent field theory describing the fluid in terms of five macroscopic or "Eulerian" field variables. The validity of these equations can be established by deriving them as averages over the (microscopic) equations of motion of the individual particles in the field. Having obtained them in this way one can ask if they can be derived from an unconstrained variational principle in the Eulerian variables. The answer, by reasoning familiar from the final paragraph of Section 2, is no.

THEOREM 4.1. There does not exist any action principle for Euler's equation which is based on the free variation of the Eulerian variables.

Proof. By Theorem 2.2, if there were such a principle then any stationary state of the fluid (in which all the Eulerian variables were time- independent) would represent an extremum of the total energy. One can give three distinct counterexamples to this.

(a) The energy of an isolated fluid at rest can be changed to first order by adding heat (entropy).

(b) The energy of such a fluid can be changed to first order by adding a particle.

(c) The energy of a uniformly flowing fluid (such as a rotating star) can be changed to first order by increasing its velocity in the direction of the original flow. 
As in electromagnetism, the only way to construct an unconstrained variational principle is to introduce potentials, some of which in the fluid case, must be nonstationary even for stationary states. This is the fundamental reason for the usefulness of such potentials in the formulations of van Dantzig [46], Eckart [10], Seliger and Whitham [37], Schmid [31] (and references therein), and Schutz [34]. (For an explicit example of the nonstationary potentials for the stationary, rotating star see Schutz $[35,36]$. Although the nonstationary potentials can have physical interest (see Schmid [31] for the remarkable example of the thermasy, a potential which is defined in our Appendix), it seems to us that in many situations it is preferable from a physical point of view to apply the constraints directly. Accordingly, we now discuss the constraints implied by the three counterexamples; then we shall construct a successful variational principle in the Eulerian variables that embodies only those constraints.

\section{(b) Discussion of the Necessary Constraints}

Since counterexample (a) clearly relates to conservation of entropy, we must expect to constrain the variations to preserve entropy in some rashion. Counterexample (b) is of course not surprising either: our variations will have to preserve the number of particles. The meaning of the velocity counterexample (c) is not immediately obvious; the best way to discover its significance is to ignore it and see what happens.

For the moment, let us consider only "cold" fluids, for which $S=0$, in order to simplify the following discussion as much as possible. We therefore wish to construct a variational principle whose variations are constrained only to preserve the number of particles. We shall confine ourselves to the Newtonian problem, again for conceptual simplicity. As a Lagrangian we adopt

$$
\mathfrak{L}=\frac{1}{2} \rho_{0} v^{2}-u\left(\rho_{0}\right),
$$

where $u$ is the fluid's internal energy density and $\rho_{0}$ the matter density. As the constraint we take

$$
\delta\left[\partial_{t} \rho_{0}+\partial_{i}\left(\rho_{0} v^{i}\right)\right]=0,
$$

guaranteeing no local creation or destruction of particles by the variation. We include the constraint by using a Lagrange multiplier, that is, by varying $\rho_{0}$ freely in the new Lagrangian

$$
\begin{aligned}
\mathfrak{L}^{\prime} & \equiv \frac{1}{2} \rho_{0} v^{2}-u\left(\rho_{0}\right)+\lambda\left[\partial_{t} \rho_{0}+\partial_{i}\left(\rho_{0} v^{i}\right)\right] \\
& =\frac{1}{2} \rho_{0} v^{2}-u\left(\rho_{0}\right)-\rho_{0} \partial_{t} \lambda-\rho_{0} v^{i} \partial_{i} \lambda+\text { total time-derivative + divergence. }
\end{aligned}
$$

Throwing away the time-derivative and divergence, we find the variation

$$
\delta \mathscr{L}^{\prime}=\left(\frac{1}{2} v^{2}-\left(d u / d \rho_{0}\right)-\partial_{t} \lambda-v^{i} \partial_{i} \lambda\right) \delta \rho_{0}+\rho_{0}\left(v_{i}-\partial_{i} \lambda\right) \delta v^{i} .
$$

The velocity variation implies

$$
v_{i}=\partial_{i} \lambda \Rightarrow \boldsymbol{\nabla} \times \mathbf{v}=0 .
$$


From the density variation, recalling that $d u / d \rho_{0}=h$ (the specific enthalpy), we get

$$
\partial_{t} \lambda+v^{i} \partial_{i} \lambda-\frac{1}{2} v^{2}+h=0 .
$$

Taking the gradient of this, and using (4.7), we find

$$
\epsilon_{t} v^{i}+\nabla_{i}\left(h+\frac{1}{2} v^{2}\right)=0,
$$

which is Euler`s Eq. (4.4) for irrotational flow in the absence of gravity.

So by imposing only conservation of particles as a constraint we have come upon a variational principle that excludes rotational flow. Since this is too restrictive, the class of variations we have allowed is too large, and we do need another constraint. The nature of this constraint becomes apparent if we compare the picture of the fluid as a macroscopic flow to the picture of its as a microscopic collection of particles. In Hamilton's principle the variation is set to zero at the initial and final times. On the microscopic view this means keeping the initial and final positions of all the particles fixed (keeping the endpoints of each particle's world line fixed). But with the constraint (4.6), the macroscopic variables $\rho_{0}$ and $\mathbf{v}$ are still the basic variables, and fixing them at the initial and final times is a weaker constraint than fixing the microscopic positions of particles. There exists a very large class of variations in the particle positions that lcave the number density unchanged. It is a two-dimensional subgroup of the group of infinitesimal spatial "coordinate transformations," which we may call particle permutations. By requiring the action to be an extremum against independent permutations of the initial and final parts of the world lines we have obtained (4.7), in which we have clearly lost two degrees of freedom in the velocity field. We may guess, therefore, that the appropriate additional constraint is to permit only variations that leave unchanged the initial and final positions of the microscopic particles. In the next section we will show that this constraint does lead to the correct equations.

This constraint was used by Taub [41] and the appropriate Lagrange-multiplier form of it was first suggested by C. C. Lin in the 1950s (cf. [28, 39]), apparently with an interpretation similar to ours. However, we know of no other proof that a variational principle must incorporate it. Notice that for a quantum Bose fluid the permutations that we have just discussed are indistinguishable from the identity transformation, and so in this case it would be appropriate to demand only the constraint (4.6). This leads immediately to the well-known irrotational flow of such a fluid, Eq. (4.7). We discuss this in more detail in Section 5.

\section{(c) The Minimally Constrained Variational Principle}

We return to a relativistically invariant notation and to fluids with entropy. The constraint on the variation of the particles' world lines can be conveniently introduced by defining a vector field $\xi^{\alpha}$, which moves a world line from its "old" position in spacetime to its "new" one. Our constraint requires that this vector, which is called the Lagrangian displacement vector, vanish at the initial and final times. We definc the "Eulerian variation" $\delta Q$ of any quantity $Q$ to be its variation "in place," i.e., at 
fixed coordinate values, and the Lagrangian variation $\Delta Q$ to be the variation following the particle as it is displaced. Their precise relationship is

$$
\Delta=\delta+\underset{\xi}{\mathfrak{E}}
$$

where $\underset{\xi}{£}$ is the Lie-derivative. This is discussed more fully in Section $6 .{ }^{15}$ In our case, the preservation of entropy constraint is

$$
\Delta S=0 .
$$

In order to express the number-conservation constraint most economically, we introduce the flux vector-density

$$
\mathfrak{N}^{\alpha} \equiv n(-g)^{1 / 2} U^{\alpha}
$$

Conservation of particles then becomes

$$
\Delta \mathfrak{N}^{\alpha}=\mathbf{0}
$$

A word on the meaning of (4.10) would be appropriate here. It can be rewritten as

$$
\delta \mathfrak{N}^{\alpha}=-\underset{\xi}{\mathfrak{E}} \mathfrak{N}^{\alpha}
$$

whose divergence can be shown to be

$$
\partial_{\alpha} \delta \mathfrak{N}^{\alpha}=-\underset{\xi}{\mathfrak{f}}\left(\partial_{\alpha} \mathfrak{N}^{\alpha}\right)
$$

Similarly, Eqs. (4.8) and (4.10) show that

$$
\delta\left[\mathfrak{N}^{\alpha} \partial_{\alpha} S\right]=-\mathfrak{\xi}_{\xi}\left(\mathfrak{P} \alpha \partial_{\alpha} S\right) .
$$

The first (second) equation vanishes for all $\xi^{\alpha}$ if and only if $\partial_{\beta} \mathfrak{Y}^{\beta}=0\left(\mathfrak{l}^{\alpha} \partial_{\alpha} S=0\right)$ which is the conservation equation for particles (entropy), in the unvaried system. In other words we do not need to assume $\partial_{\beta} \mathfrak{N}^{\beta}=0$ and $\mathfrak{N}^{\alpha} \partial_{\alpha} S=0$. We permit, a priori, sources and sinks in the fluid. Our constraint says only that these are carried along by $\xi^{\alpha}$. The variation does not add or remove particles or entropy by itself. In this respect we impose only what our counterexamples in Theorem 4.1 force on us: our variational principle is minimally constrained. Our carefulness now in this respect will reward us when we discuss the extremal properties of the perfect fluid in Section 6 .

Now we must choose the appropriate Lagrangian. It is well known that if our fluid were composed of pressureless "dust," the appropriate Lagrangian would be minus the energy density, $\rho(-g)^{1 / 2}$. Moreover, if this dust were collisionless with random velocities, then the macroscopic fluid would have an average kinetic pressure, but the

${ }^{15}$ Of course in general relativity the distinction between $\delta$ and $\Delta$ is only one of "gauge." We warn the reader that in Section 6 we shall use $\delta$ in a somewhat more general sense; but in this part it always means "change in place." 
Lagrangian would still be the same. This suggests that the Lagrangian of a general fluid with pressure should still be

$$
\mathfrak{Q}=-\rho(-g)^{1 / 2}
$$

That this reduces to (4.5) in the nonrelativistic limit can be proved as follows. In the comoving frame we write

$$
\rho(-g)^{1 / 2} d^{1} x=\rho d V d s=\left(\rho_{0}+u\right) d V d s
$$

where $d \boldsymbol{l}$ is the comoving volume element and $s$ is the proper time, and where we have decomposed $\rho$ into rest mass and internal energy measured in the comoving frame. In a frame in which the fluid has velocity $v$, the time-coordinate $t^{\prime}$ satisfies

$$
d s=\left(1-v^{2}\right)^{1 / 2} d t^{\prime}=\left(1-\frac{1}{2} v^{2}\right) d t^{\prime}+O\left(v^{4}\right) .
$$

Now, $\rho_{0} d V$ is the total rest-mass $d M_{0}$ in the element, which is the same in all frames, and $d V^{\prime} d s \cdots d V^{\prime \prime} d t^{\prime}$ is the invariant volume element, where $d V^{\prime}$ is the three-volume in the new frame. So in that frame one has

$$
\rho(-g)^{1 / 2} d^{4} x=d M_{0}\left(1-\frac{1}{2} v^{2}\right) d t^{\prime}+u d V^{\prime} d t^{\prime}+O\left(v^{4}\right) .
$$

Since $d M_{0} d t^{\prime}$ is a constant under the variations, it can be discarded, and we find

$$
\rho(-g)^{1 / 2} d^{4} x \rightarrow\left(u-\frac{1}{2} \rho_{0} v^{2}\right) d V^{\prime} d t^{\prime}+O\left(v^{4}\right)
$$

Now to the variations of $\mathfrak{L}$. We wish to vary

$$
S=\int 2 d^{4} x
$$

according to the constrained $\delta \mathfrak{N}^{\alpha}$ and $\delta S$, holding the metric fixed

$$
\delta g_{\alpha \beta}=0 \Leftrightarrow \Delta g_{\alpha \beta}=\underset{\xi}{£} g_{\alpha \beta}=2 \xi_{(\alpha ; \beta)}
$$

where round brackets denote symmetrization. Since $\xi^{\alpha}$ vanishes on the boundary of the region, it does not matter whether we use $\delta$ or $\Delta$ inside. For reasons of simplicity we take

$$
\delta S=\int \Delta \mathfrak{L} d^{4} x
$$

Since the variation $\Delta$ holds $\mathfrak{Y}^{\alpha}$ fixed, it varies $n$ through $\Delta g_{\alpha \beta}$ according to the expression

$$
n=\left(\mathfrak{N}^{\alpha} \mathfrak{M}^{\beta} g_{\alpha \beta} / g\right)^{1 / 2}
$$

where $g$ is the determinant of $g_{x \beta}$. From this we find

$$
\Delta n=-\frac{1}{2} n q^{\alpha \beta} \Delta g_{x \beta},
$$


where we have used the projection tensor orthogonal to $U^{\alpha}$,

$$
q^{\alpha \beta} \equiv g^{\alpha \beta}+U^{\alpha} U^{\beta} .
$$

By the first law of thermodynamics,

$$
d \rho=\mu d n+n^{\top} T d S
$$

we have

$$
\Delta \rho=\mu \Delta n
$$

Adding to this the identity

$$
\Delta(g)^{1 / 2}=\frac{1}{2}(g)^{1 / 2} g^{\alpha \beta} \Delta g_{\alpha \beta}
$$

we easily find

$$
\Delta\left(\rho(-g)^{1 / 2}\right)=-\frac{1}{2} T^{\alpha \beta}(-g)^{1 / 2} \Delta g_{\alpha \beta},
$$

where $T^{\alpha \beta}$ is the correct tensor for the perfect fluid, (4.2). This equation of course has to hold if $\mathfrak{E}$ is the correct Lagrangian. What is more, in our case it can be combined with (4.11) and (4.12) to give

$$
\delta S=-\int \xi^{\alpha} \nabla_{\beta} T_{\alpha}^{\beta}(-g)^{1 / 2} d^{4} x,
$$

where an integration by parts has been performed and the integrated term discarded because $\xi^{\alpha}$ vanishes on the boundary. We have therefore established that all and only those configurations satisfying $\nabla_{\alpha} T_{\beta}^{\alpha}=0$ are extrema of the action against all variations that obey the constraints (4.8) and (4.10).

It is important to notice that the equation of continuity is not one of the EulerLagrange equations. The only thing implied is

$$
-U^{\alpha} \nabla_{\beta} T^{\beta}{ }_{\alpha}=\mu \nabla_{\alpha}\left(n U^{\alpha}\right)+n T U^{\alpha} \partial_{\alpha} S=0,
$$

which, from Eq. (4.15), is often called the conservation of energy equation. A welldetermined system requires some additional, ad hoc specification of the nature of particle creation. Conventionally no creation is allowed, but one could equally possibly allow some (e.g., by counting numbers of free particles rather than baryons in nuclear reactions). Then (4.16) says that the entropy per particle has to change in order to keep the total energy fixed, since heat flow is forbidden by the form of $T^{\alpha \beta}$. In any case, once such a law is given, the correct solution extremizes the action among configurations that obey the same law.

It is a remarkable feature of this variational principle that the equations of motion can be derived via the stress-energy tensor, that is, only using $\Delta g_{\alpha \beta}$. More commonly field equations are derived directly from the variations of the dynamical fields; then, as in Section 2, the stress-energy tensor is derived by variation of the metric. The scalar nature of the Lagrangian proves that the field equations always imply the vanish- 
ing of the covariant divergence of $T_{\beta}^{\alpha}$ but not necessarily vice versa. What makes the perfect fluid special is that its constraint is that $\delta$ be induced by a Lie-dragging: requiring the action to be stationary against a Lie-dragging in which the metric is not dragged (the conventional way one would produce the equations of motion) is equivalent for a scalar Lagrangian to requiring the action to be stationary against a Lie-dragging in which the metric is dragged but the field variables are not (the usual way of deriving conservation of $T^{\alpha \beta}$ ). Any constrained variational principle with a scalar Lagrangian in which the constraint allows only an arbitrary Lie-dragging as a variation will produce field equations completely equivalent to the equations $\nabla_{\alpha} T_{\beta}^{\alpha}=: 0$. where $T_{B}^{\alpha}$ is the stress-energy tensor of that field.

This variational principle is closely related to one given by Hawking and Ellis [15] who ignore entropy and impose conservation of particles before the variations are made. The original variational principle of this sort in relativity was, as far as we can determine, given by Taub [42], who employed some Lagrange multipliers. A version of our variational principle that eliminates constraints with Lagrange multipliers is given in the Appendix, with a discussion of its relation to velocity-potential Lagrangians.

\section{Superfluid Fiow}

Before applying our "minimally constrained" variational principle to the study of energy extrema in fluids, we pause to point out the physical meaning of the unconsstrained ${ }^{16}$ principle, which in Section $4 \mathrm{~b}$ (Eq. 4.5) we introduced as no more than a foil to be abandoned immediately in favor of the "minimally constrained" principle of Section 4c. We show in fact that the unconstrained principle is precisely that suited to describe the zero-entropy motions of a degenerate bose-fluid. In this way we provide a "deep" reason for the well-known irrotational character of superfluid flow.

According to the path-integral approach to quantum mechanics, the amplitude for a particle at event $x$ to arrive at event $y$ is the integral over all conceivable paths from $x$ to $y$ of an amplitude proportional to $e^{i S / \hbar}$ where $S$ is the classical action associated with the path. The classical law of motion, $\delta S=0$, amounts here to the observation that, in the stationary phase approximation, only such a motion contributes to the

\footnotetext{
${ }^{16}$ Even in this principle, which we are calling in this section "unconstrained," the variations are restricted by the conservation of matter, Eq. (4.6). We feel however that this constraint is inessential in a way in which the "preservation of particle identity" constraint (Section $4 b$ ) is not. One symptom of this, which perhaps could serve to define "inessentiality," is that the unperturbed version of Eq. (4.6) can be solved locally by expressing $\rho$ and $v$ in terms of potentials through which Eq. (4.6) becomes an identity $\rho=\boldsymbol{\nabla} \cdot \mathbf{A}, \rho \mathbf{v}=-\dot{\mathbf{A}}+\boldsymbol{\nabla} \times \mathbf{B}$. If we allow ourselves constraints on the unperturbed fields $\rho, \mathbf{v}$ (but only constraints soluble in this, or in some more appropriate, sense), then we can treat the variations $\delta \rho, \delta \mathrm{v}$ as unconstrained within the class of allowable unperturbed fields. The identity of particles constraint, in contrast, is a restriction on the variations per se, because the class of allowed perturbations is of necessity smaller than that of unperturbed fields. We would conjecture that it is only constraints of this "essential" kind which invalidate the results derived for unconstrained principles in Section 2.
} 
path integral. Furthermore, by choosing a suitable initial superposition of amplitudes one can construct a wave-packet which actually follows the classical path, although the uncertainty principle implies an inevitable smearing of the classical world line over a radius, at temperature $T$, of about

$$
\Delta x \sim \hbar / p \sim \hbar /(m k T)^{1 / 2} .
$$

Consider now a fluid composed of identical bosons. As long as the interparticle distance is much greater than this "degeneracy length," $\Delta x$, the particles will behave (at least between collisions) like distinct individuals, and will have classical paths in the same sense as an isolated particle does. But when the fluid is so dense that the wavepackets must overlap, such as in $\mathrm{He}^{4}$ below the lambda-point, a description in terms of separate particle paths no longer makes sense.

In the path integral terms the amplitude to go from a configuration with particles at $x_{1} \cdots x_{N}$ to one with particles at $y_{1} \cdots y_{N}(N=$ number of particles in the system) is a combination of sums, not only over all motions which take a particle at $x_{1}$ to $y_{1}$, a particle at $x_{2}$ to $y_{2}$, etc., but also over all motions which take particles at $x_{1} \cdots x_{N}$ to $y_{1} \cdots y_{N}$ in any permutation. (For bosons all terms occur with the same sign.) $\Lambda$ s before we can argue that only motions which make the total action, $S$, stationary will contribute to the sum. When the fluid exceeds the degeneracy density, not only the usual deformation of world lines with endpoints fixed but also a permutation of the endpoints themselves represents an infinitesimal variation of the overall path, against which $S$ must therefore be stationary. We conclude that the coherent motions of a degenerate bose fluid correspond to motions which extremize the action against all variations of the particle world lines, including variation of the endpoints. ${ }^{17} \mathrm{In}$ other words, the naive Eulerian approach which led to Eq. (4.7) is actually correct for degenerate bose fluids, and yields motions for which the fluid as a whole is in a single quantum state..$^{18}$ In particular, by Eq. (4.7), the flow is irrotational. ${ }^{19}$

${ }^{17}$ Subject, of course, to appropriate boundary conditions, which for a fluid in a vessel say that $\delta(\rho v)$ is transverse to the walls and that $\delta \rho=0$ at the initial and final times.

${ }^{18}$ By adapting the Eulerian approach to the two-fluid model of liquid helium one produces automatically the correct phenomenological equations. This has been long known but never understood $[19$, Section 19]. Notice that since the entropy bearing component of $\mathrm{He}(\mathrm{II})$, the so-called normal fluid, represents a nondegenerate gas of phonons and rotons, variations with fixed world line endpoints are appropriate to it. Because London ignores this constraint he gets field equations which are too restrictive. In particular, Eq. (9) of Section 19 would rule out, say, rigid rotation even of the normal fluid, which is silly. Despite this error, the equations of motion $\left(12^{\prime}\right)$ and $\left(13^{\prime}\right)$, derived by eliminating the potentials $\alpha$ and $\beta$, are correct.

${ }^{19}$ If we accept, as argued in the first footnote in this section, that Theorem 2.2 should apply in the presence of "inessential" constraints, then we can see directly why the flow must be irrotational. Otherwise, there would be stationary motions (e.g. rotations) not extremizing the total energy, even though conservation of mass is the only constraint. In the present case we can make the argument precise by (1) noting that Theorem 2.2 applies immediately if we choose the potentials mentioned in the first footnote as basic variables, and (2) noticing that these potentials can be chosen stationary when the fluid flow itself is stationary. By contrast, the potentials which one introduces to eliminate the particle-identity constraint (as described in the Appendix) cannot be chosen stationary for stationary flows. This is no doubt due to the "essentiality" of that constraint. 
The relativistic version of Eq. (4.7) can be worked out by varying $\mathfrak{N}^{\mu}$ in the Lagrangian (4.11) subject only to conservation of mass

$$
\partial_{\mu} \mathfrak{N}^{\mu}=0 .
$$

The equations of motion (found just as in Section $4 b$ ) are

$$
\nabla_{\mu} V_{\nu}=\nabla_{\nu} V_{\mu}
$$

where

$$
V_{\mu}=((\rho+p) / n) U_{\mu} .
$$

It is thus $V_{\mu}$ rather than the four-velocity $U_{\mu}$ which is irrotational, and the four components of this relation constitute the entire equations of motion. It is possible that (5.1)-(5.3) describe conditions inside certain neutron stars [30].

\section{Extremal Theorems for the Perfect Fluid}

In this section we make the extension of Section 2 to the case of the perfect fluid. The constraints on the variations of the field variables for the perfect fluid show up in the extremal theorems as restrictions on the class of perturbations which leave the energy unchanged to first order. But the theorems we are able to prove turn out to apply to any stress-energy tensor, without restriction on whether it is derivable from a Lagrangian at all. Besides the insight that these extremal theorems give into the structure of conservation laws, they have considerable interest in astrophysics because they lead to useful variational principles for the structure of stationary configurations (Theorem 6.4 below). Moreover, they are a necessary prerequisite for a discussion of the stability of solutions, because stability concerns the second-order changes in energy, which are only welldefined if the first-order changes vanish. All the results of this section are derived for general relativity, but the Newtonian versions of the more important theorems are also given.

\section{(a) Killing Vectors and Conserved Quantities}

A discussion of the extremal properties of perfect-fluid solutions must start with the analog of Eq. (2.16), which we now derive. We consider a variation $\underset{\xi}{\delta}$ that drags everything along $\xi$, including the boundary of the region of integration. This amounts to an infinitesimal "change in coordinates," which leaves the action invariant.

The action has two pieces

$$
\begin{aligned}
S & =S_{G}+S_{F}, \\
S_{G} & =(1 / 16 \pi) \int_{\mathfrak{X}} R(-g)^{1 / 2} d^{4} x, \\
S_{F} & =-\int_{\mathfrak{X}} \rho(-g)^{1 / 2} d^{4} x .
\end{aligned}
$$


In Section 4 we considered a variation of $S_{F}$ which consisted of a dragging of the fluid variables holding the metric and boundary fixed. This produced a change

$$
-\int_{\mathfrak{X}} \mathfrak{T}_{\nu ; \mu}^{\mu} \xi^{\nu} d^{4} x
$$

in $S_{F}$. To this we add the change due to the dragging of the metric holding the boundary fixed, plus the change due to the variation at the boundary, which defines the fluid's Noether operator by analogy with Eq. (2.4) of the unconstrained case. The result is

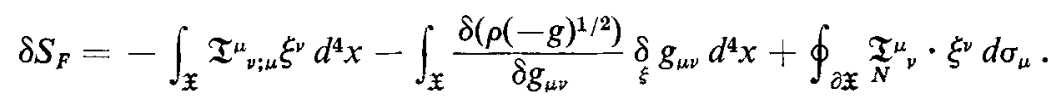

Since the symmetric stress-energy tensor is just

$$
\mathfrak{T}^{\mu \nu}=-2\left(\delta\left(\rho(-g)^{\mathbf{1 / 2}}\right) / \delta g_{\mu \nu}\right),
$$

the fact that $\underset{\xi}{\delta} S_{F}$ vanishes implies (sincc $\underset{\xi}{\delta} g_{\mu \nu}=-\underset{\xi}{f} g_{\mu v}=-2 \xi_{(\mu ; v)}$ )

$$
\oint_{\partial \mathfrak{X}} \frac{\mathfrak{T}}{N}_{\nu} \cdot \xi^{\nu} d \sigma_{\mu}=\oint_{\partial \mathfrak{X}} \mathfrak{I}^{\mu}{ }_{\nu} \xi^{\nu} d \sigma_{\mu}
$$

Since the equations of motion of the fluid have not been imposed, this equation is an identity and shows that the symmetric $\mathfrak{I}^{\mu}{ }_{\nu}$ is a Noether operator for the fluid. Using this in Eq. (6.1), we get the identity

$$
\begin{aligned}
\delta_{\xi} S_{F}= & =-\frac{1}{2} \int_{\mathfrak{X}} \mathfrak{I}^{\alpha \beta} \underset{\xi}{\mathfrak{f}_{\xi \alpha \beta}} g_{\alpha} d^{4} x-\int_{\mathfrak{X}} \mathfrak{T}^{\alpha \beta}{ }_{; \beta} \xi_{\alpha} d^{4} x \\
& +\oint_{\partial \mathfrak{X}} \mathfrak{T}^{\alpha \beta} \xi_{\alpha} d \sigma_{\beta} .
\end{aligned}
$$

We must add to this the variation of $S_{G}$ from Section 3

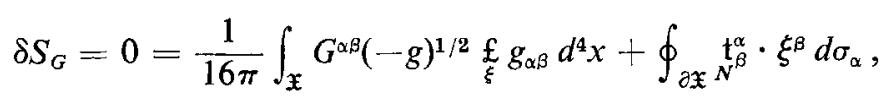

where $\mathfrak{t}_{N^{\beta}}^{x}$ is the gravitational Noether operator appropriate to the kind of motion generated by $\xi$ (as alluded to in Section 3). Combining this with (6.2) gives

$$
\begin{gathered}
-\frac{1}{16 \pi} \int_{\mathfrak{X}}\left(G^{\alpha \beta}-8 \pi T^{\alpha \beta}\right)(-g)^{1 / 2}{\underset{\xi}{\mathfrak{E}}}_{g_{\alpha \beta}} d^{4} x+\int_{\mathfrak{X}} \mathfrak{T}_{\beta ; \alpha}^{\alpha} \xi^{\beta} d^{4} x \\
=\oint_{\partial \mathfrak{X}}\left(\mathrm{t}_{\beta}^{\alpha} \cdot \xi^{\beta}+\mathfrak{T}_{\beta}^{\alpha} \xi^{\beta}\right) d \sigma_{\alpha} .
\end{gathered}
$$

This is the key equation for the problem at hand. We have so far made no assumptions about $\xi^{\alpha}, g_{\alpha \beta}$, or the fluid variables. In fact, Eq. (6.3) is an identity for any stressenergy tensor, not just one describing a perfect fluid, and so many of our results will apply to general systems. What makes this equation particularly powerful in the case of the perfect fluid is that the Einstein equations,

$$
G^{\alpha \beta}-8 \pi T^{\alpha \beta}=0
$$


completely determine the dynamics of the system. No extra dynamical equations for the source of the metric are required because the complete equations for the fluid are

$$
T_{; \beta}^{\wedge \beta}=0 \text {, }
$$

which are a direct consequence of the Einstein equations. This special situation follows, as discussed in Section 4, from the fact that the constraints permit only Lie-draggings. Since these constraints are therefore respected by the variations that led to (6.3), this equation will be particularly well suited to the fluid problem.

The total $\xi$-momentum for an asymptotically regular hypersurface and vector field $\xi$ (as defined in Section 3 ) is

$$
P[\xi, \mathscr{H}]=\int_{\mathscr{H}}\left(\mathrm{t}_{N}^{\mu} v \cdot \xi^{\prime \prime}+\mathfrak{I}^{u}{ }_{\nu} \xi^{\nu^{\prime}}\right) d \sigma_{\mu}
$$

If the initial-value equations are satisfied on $\mathscr{H}$, then $P[\xi, \mathscr{H}]$ depends only on the asymptotic behavior of $\xi^{\mu}$ and the metric, and so (with appropriate $\xi^{\mu}$ and $\mathfrak{t}_{N}^{\mu}$ ) determines the total energy, linear momentum, and angular momentum of the spacetime as measured by the orbits of particles at spatial infinity. But $P[\xi, \mathscr{H}]$ may be calculated for any fields, regardless of whether they satisfy the initial-value equations or not (and in Section 3 we have verified, for the case of four-momentum, that it is independent of the particular asymptotic behavior of the fields). Therefore we shall use it to define the $\xi$-momentum of any metric and matter field. Equation (6.3) then gives the following result.

Proposition 6.1. Let an asymptotically regular vector field be defined everyuhere on a manifold that contains matter of compact spatial support. ${ }^{20}$ Then the total $\xi$ momentum on any asymptotically regular hypersurface $\mathscr{H}$ is conserved (independent of $\mathscr{H})$ if either

(a) the metric and matter satisfy Einstein's equations; or

(b) $\xi^{\alpha}$ is a Killing vector of the metric, and the matter's $\xi$-momentum is locally. conserved

$$
\partial_{\beta}\left(\xi^{\alpha} \mathfrak{I}_{\alpha}^{\beta}\right)=0 .
$$

Proof. (a) By hypothesis the first term on the left of Eq. (6.3) vanishes; then the Bianchi identities imply that the second term also vanishes. By arguments used in Section 3 it follows that $P[\xi, \mathscr{H}]$ does not depend on $\mathscr{H}$. (h) The two assumptions make the left side of (6.3) vanish, since we have

$$
\partial_{\beta}\left(\xi^{\alpha \mathfrak{I}^{\beta}}{ }_{\alpha}\right)=(-g)^{1 / 2}\left(\xi_{\alpha} T^{\alpha \beta}\right)_{; \beta}=(-g)^{1 / 2} \xi_{\alpha} T_{; \beta}^{\alpha \beta}
$$

by virtue of the fact that $\xi$ is a Killing vector. This again implies conservation of $P[\xi, \mathscr{H}]$ by arguments familiar from Section 2 .

${ }^{20}$ As in Theorem 2.2 the compactness restriction is merely a convenience. Massless fields of finite total $\xi$-momentum can also be included without changing the proposition. 
This proposition is, again, fully general. It does not require a variational principle for the matter fields.

The Newtonian version of these results is somewhat more complicated because of the split between time and space. We shall restrict ourselves to perfect fluids and consider only the energy. In the notation of Section 4, the analog of Eq. (6.3) is

$$
\begin{aligned}
\int_{t_{1}}^{t_{2}} d t \int & d^{3} x\left\{\left(-\frac{1}{4 \pi} \nabla^{2} \Phi+\rho_{0}\right) \partial_{t} \Phi+n T\left(\partial_{t} S+\mathbf{V} \cdot \nabla S\right)\right. \\
& +\left[\partial_{t} \rho_{0}+\nabla \cdot\left(\rho_{0} \mathbf{V}\right)\right]\left(\Phi+\frac{1}{2} V^{2}+h\right) \\
& \left.+\rho_{0} \mathbf{V} \cdot\left[\partial_{t} \mathbf{V}+(\mathbf{V} \cdot \nabla) \mathbf{V}+\nabla \Phi+\frac{1}{\rho_{0}} \nabla p\right]\right\} \\
= & {\left[\int d^{3} x\left(U+\frac{1}{2} \rho_{0} V^{2}+\rho_{0} \Phi+\frac{1}{8 \pi} \nabla \Phi \cdot \nabla \Phi\right)\right]_{t_{1}}^{t_{2}}, }
\end{aligned}
$$

where the notation $[A]_{t_{1}^{2}}^{t_{2}}$ means $A\left(t_{2}\right)-A\left(t_{1}\right)$. This equation is an identity apart from discarding a spatial divergence, which vanishes if the integral over $d^{3} x$ is taken over all space. A certain amount of algebra establishes the following result.

PROPOSITION 6.1N. The energy of a fluid in a gravitational field,

$$
E=\int d^{3} x\left(U+\frac{1}{2} \rho_{0} V^{2}+\rho_{0} \Phi+(1 / 8 \pi) \nabla \Phi \cdot \nabla \Phi\right),
$$

is conserved if either

(a) the fluid and field satisfy Newton's and Euler's equations plus the entropy and mass conservation equations; or

(b) the gravitational field is time independent and the energy of the fuid is locally conserved

$$
\partial_{t}\left(U+\frac{1}{2} \rho_{0} V^{2}+\rho_{0} \Phi\right)+\nabla \cdot\left[\left(\frac{1}{2} V^{2}+h+\Phi\right) \rho_{0} \mathbf{V}\right]=0 . \quad \square
$$

(b) Definition of a Perturbation

As in Section 2, much more information can be extracted from Eq. (6.3) by perturbing it. We shall find it useful to define carefully the notion of a perturbation. We begin with an unperturbed manifold $M$ upon which various fields $\{Q\}$ are defined. A perturbation of $M$ consists of a perturbed manifold $M^{\prime}$ containing fields $\left\{Q^{\prime}\right\}$, plus a 1-1 map, $e$, from $M^{\prime}$ onto $M^{21}$ This map generates unique maps of the fields $\left\{Q^{\prime}\right\}$ into fields $\left\{Q_{*}\right.$ ' $\}$ on $M$. We are only interested in "small" perturbations, which are those for which $\left\{Q_{*}{ }^{\prime}\right\}$ and $\{Q\}$ differ only "infinitesimally." We denote by $\delta_{e} Q$ the difference $Q_{*}{ }^{\prime}-Q$ in $M$. We work only to first order in $\delta_{e} Q$, so that $\delta_{e}$ is a derivation.

${ }^{21}$ It may not always be necessary or possible to map all of $M^{\prime}$ onto $M$, for instance if the perturbation grows to develop a horizon and/or a singularity in $M^{\prime}$ that does not exist in $M$. What we really require is a map of an open region of $M^{\prime}$ that contains a Cauchy hypersurface onto a similar region of $M$. 
The map $e$ is, of course, to a large extent arbitrary; for infinitesimal perturbations any infinitesimal change in $e$ to a new map $\int$ will leave the perturbations $\delta_{f} Q$ infinitesimal. Such a change defines a vector field $\eta$ in $M$, which carries the image of a point under $e$ into its image under $f$. For infinitesimal changes we clearly have

$$
\delta_{f}=\delta_{e}-\underset{\eta}{\mathfrak{f}}
$$

Sometimes there are physical reasons for choosing a certain map. In fluid dynamics one defines (as we did in Section 4) the Lagrangian (or comoving) map $l$ to carry the world line of a particle in $M^{\prime}$ into that of the "same" particle in $M$. The derivation $\delta_{l}$ induced in $M$ is usually called $\Delta$. Other maps may also be useful, especially outside the fluid, where $l$ is undefined. If $M^{\prime}$ and $M$ are isometric (as in Newtonian gravity) one may want $e$ to be an isometry.

This discussion has so far not mentioned coordinates on $M$ and $M^{\prime}$, and is therefore suitable only when the fields $Q$ are tensors or tensor densities. In our work below we shall have to perturb not only tensor fields but also the integral of the gravitational Noether operator, $\mathfrak{t}_{v^{\prime}}^{\mu}$. By Eq. (3.7) however, this integral consists of the integral of the vector density $G_{\nu}^{\mu} \xi^{\nu}(-g)^{1 / 2}$ over a hypersurface plus a two-surface integral of the coordinate-dependent divergence term in the asymptotically flat region. We shall always choose our map $e$ to preserve the coordinates in the asymptotic region, but leave the coordinates free elscwherc. In nonrelativistic language, the perturbation operator $\delta_{\rho}$ is Eulerian at infinity but quite general elsewhere. We shall from now on drop the subscript $e$ on $\delta_{e}$, but it is important for our later results to realize that $\delta$ is not necessarily what is conventionally called an Eulerian perturbation.

\section{(c) General Extremal Theorem for General Relativity}

We consider a region $\mathfrak{t}$ of a manifold with an arbitrary metric, containing a perfect fluid, and upon which an arbitrary vector field $\xi$ is defined. We allow the perturbation to he arbitrary (but infinitesimal), and we define a region $\mathfrak{x}^{\prime}$ of $M^{\prime}$ and a field $\xi^{\prime}$ on $M^{\prime}$ such that $e\left(\mathfrak{X}^{\prime}\right)=\mathfrak{X}$ and $\delta \xi=0$ (which latter condition implies that $\delta$ and $£$ commute on $M$ ). The first variation of Eq. (6.3) gives the identity

$$
\begin{aligned}
& \int_{X} \int_{-1}-\frac{1}{16 \pi}\left(G^{\alpha \beta}-8 \pi T^{\alpha \beta}\right)(-g)^{1 / 2} \underset{\xi}{£} \delta g_{\alpha \beta}-\frac{1}{16 \pi} \delta\left[\left(G^{\alpha \beta}-8 \pi T^{\alpha \beta}\right)(-g)^{1 / 2}\right] \underset{\xi}{f} g_{x \hbar}
\end{aligned}
$$

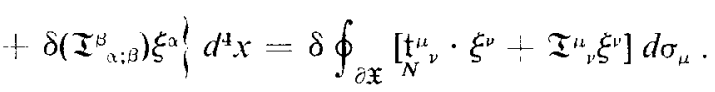

As before, the vanishing of the right-hand side implies conservation of the first variation of the integral $\xi$-momentum. We are concerned to determine the conditions under which this can happen. The analog of Theorem 2.2 is the following.

THEOREM 6.2. Define $P[\xi, \mathscr{H}]$ for asymptotically regular $(\xi, \mathscr{H})$ by Eq. (6.3). Of the following, any two imply the third

(a) the metric is stationary with respect to $\xi: £ g_{\alpha \beta}=0$; 
(b) the Einstein equations are satisfied: $G^{\alpha \beta}-8 \pi T^{\alpha \beta}=0$ (with the implication $T_{; \beta}^{\alpha \beta}=0$ ) wherever $\xi \neq 0$ :

(c) $P[\xi, \mathscr{H}]$ is an extremum for fixed $\xi$ and any $\mathscr{H}$ against all variations of the metric and matter fields that vanish on some. $\mathscr{H}^{\prime}$ and satisfy

$$
\delta\left(\mathfrak{T}_{\alpha ; \beta}^{\beta}\right) \xi^{\alpha}=0
$$

in the region between $\mathscr{H}$ and $\mathscr{H}^{\prime}$.

Proof. (a) + (b) $\Rightarrow$ (c). By hypothesis the first two terms on the left of Eq. (6.6) vanish. For any variation $\delta$ of the type contemplated in the theorem, condition (c) provides a region $\mathfrak{X}$ bounded by hypersurfaces $\mathscr{H}$ and $\mathscr{H}^{\prime}$ that is like that used in the proof of Theorem 2.2 and depicted in Fig. 1. (The possibility that $\mathscr{H}$ and $\mathscr{H}^{\prime}$ intersect is most easily handled by introducing a third hypersurface $\mathscr{H}^{\prime \prime}$ disjoint from them both, on which the perturbation also vanishes.) We can assume without loss of generality that $\delta$ is of compact support, since the restriction (6.7) is vacuous outside the fluid (which is itself of compact spatial support) and since we showed in Section 3 that any allowable $\delta$ is equivalent on $\mathscr{H}$ to one of compact support. Thus the entire left-hand side of (6.6) vanishes, while (just as in Section 2) the right-hand side reduces to $\delta P[\xi, \mathscr{H}]$.

(a) + (c) $\Rightarrow$ (b). Just as in the proof of Theorem 2.2, we get, for any $\mathfrak{X}$ and all allowed perturbations $\delta$,

$$
\int_{\mathfrak{X}} \mathfrak{E}^{\alpha \beta} \underset{\xi}{\mathfrak{\xi}} \delta g_{\alpha \beta} d^{4} x=0,
$$

where we have set, as before, $\mathbb{(}^{\alpha \beta}=\left(G^{\alpha \beta}-8 \pi T^{\alpha \beta}\right)(-g)^{1 / 2}$. Since Eq. (6.7) is only one condition on the variations, it can be taken to restrict the variations in the matter fields, leaving $\delta g_{\alpha \beta}$ free at every point. This implies $\mathfrak{E}^{\alpha \beta}=0$.

(b) $+($ c) $\Rightarrow$ (a). As before, we get

$$
\int_{\mathfrak{X}} \delta \tilde{\boldsymbol{E}}^{\alpha \beta} \underset{\xi}{£} g_{\alpha \beta} d^{4} x=0
$$

for all allowed pairs $(\mathfrak{X}, \delta)$. At any point the free variations $\delta g_{\alpha \beta}$ may be chosen to make all ten $\delta \mathfrak{E}^{\alpha \beta}$ linearly independent; by choosing $\mathfrak{x}$ to be a small enough neighborhood of that point one concludes that $\sum_{\xi}^{£} g_{\alpha \beta}=0$ there.

The restriction (6.7) allows this theorem to work for any matter fields, while the unrestricted Theorem 2.2 applies only to fields that have unconstrained variational principles. The price one pays for this wider scope is a less general result. The present theorem says only that the Einstein equations are satisfied (given stationarity and extremality), while Theorem 2.2 would imply the matter field equations as well. For the perfect fluid, of course, any matter field equations would be redundant, and so Theorem 6.2 is perfectly general in the case we are most interested in. 
(d) Systems with Asymptotically Timelike Killing Vectors

The most important applications of Theorem 6.2 will be to extremize of the energy of stationary systems. When the system has a killing vector the restriction. Eq. (6.7), on the variations becomes much more transparent.

LEMMA 6.3. If the unperturbed metric and stress-energy tensor are invariant under motions along $\xi^{\star}$, so that

$$
\underset{\xi}{£} g_{\alpha \beta}=\underset{\xi}{£} T_{\alpha \beta}=0,
$$

but are otherwise arbitrary, then

(a) for an arbitrary region $\mathfrak{x}$ the following is an identity

$$
\int_{\mathfrak{X}} \xi^{\alpha} \delta\left(\mathfrak{T}_{\alpha ; \beta}^{\beta}\right) d^{4} x=\oint_{\partial x}\left(\delta \mathfrak{I}_{\alpha}^{\beta}-{ }_{2}^{1} \mathfrak{I}^{\mu \nu} \delta g_{\mu \nu} \delta_{\alpha}^{\beta}\right) \xi^{\alpha} d \sigma_{b i} ;
$$

(b) if $T_{a / 3}$ is the perfect-fiuid stress-energy tensor, then the surface integral in (a) becomes

$$
\oint_{X}\left(\mathfrak{N}^{\nu} \delta V_{\nu} \delta^{\beta}{ }_{\tau}-\mathfrak{N}^{\beta} \delta V_{\alpha}-V_{\alpha} \delta \mathfrak{N}^{\beta}+n T(-g)^{1 / 2} \delta S \delta_{\S}^{\beta}\right) \xi^{\alpha} d \sigma_{f \prime},
$$

where we define

$$
V_{v} \equiv \mu U_{v},
$$

which is conventionally called the momentum per particle.

Proof. Because $\delta \xi^{\alpha}=0$ by definition, we have

$$
\begin{aligned}
& \xi^{\alpha} \delta\left(\mathfrak{T}_{\alpha ; \beta}^{\beta}\right)=\delta\left(\mathfrak{T}_{\alpha ; \beta}^{\beta} \xi^{\alpha}\right) \\
& =\delta\left[\left(\mathfrak{T}_{\alpha}^{\beta} \xi^{\alpha}\right)_{, \beta}-\frac{1}{2} \mathfrak{T}^{\alpha \beta} \sum_{\xi} g_{\alpha \beta}\right] \\
& =\delta\left[\left(\mathfrak{I}_{\alpha}^{\beta} \xi^{\alpha}\right)_{\beta}\right]-\frac{1}{2} \underset{\xi}{\mathfrak{f}}\left(\mathfrak{T}^{\alpha \beta} \delta g_{\alpha \beta}\right) \\
& =\delta\left[\left(\mathfrak{T}^{\beta}{ }_{\alpha} \xi^{\alpha}\right)_{, \beta}\right]-\frac{1}{2} C_{\alpha}\left(\mathfrak{I}^{\mu \nu} \delta g_{\mu \nu} \xi^{*}\right) \text {. }
\end{aligned}
$$

where the second step follows from the vector density nature of $\mathfrak{I}_{\alpha}^{\beta} \xi^{\star}$, the third step from the hypotheses of the lemma, and the final step from the expression for the Lie derivative of a scalar density. Moreover, since the divergence in the first term in the last equation depends only on the manifold structure, it commutes with any mapping, and so commutes with $\delta$. This then proves (a) for any invariant $T^{\alpha \beta}$. To find the form of the surface integral for the perfect fluid, we write

$$
\mathfrak{T}_{\alpha}^{3}{ }_{\alpha}=V_{\alpha} \mathfrak{N}^{8}+\delta^{e}{ }_{\alpha} p(-g)^{1 / 2} .
$$

The identity $\mu=\left(-V^{\alpha} V^{g} g_{\alpha \beta}\right)^{1 / 2}$ implies

$$
\delta \mu=-U^{\alpha} \delta V_{\alpha}+\frac{1}{2} U^{\alpha} V^{\beta} \delta g_{\alpha \beta} .
$$


One can easily use this and the first law of thermodynamics,

$$
\delta p=n \delta \mu-n T \delta S,
$$

to establish (b).

This lemma does not assume that the unperturbed Einstein equations are satisfied, and it is valid for any Killing vector. When the hypotheses of the Lemma are satisfied we also have

$$
\begin{aligned}
& \int_{\mathfrak{X}} \frac{1}{16 \pi}\left(G^{\alpha \beta}-8 \pi T^{\alpha \beta}\right)(-g)^{1 / 2} \underset{\xi}{\underset{\xi}{f} \delta g_{\alpha \beta} d^{4} x} \\
& \quad=\frac{1}{16 \pi} \int_{\mathfrak{X}} \underset{\xi}{E}\left[\left(G^{\alpha \beta}-8 \pi T^{\alpha \beta}\right)(-g)^{1 / 2} \delta g_{\alpha \beta}\right] d^{4} x \\
& \quad=\oint_{\partial X} \frac{1}{16 \pi}\left(G^{\alpha \beta}-8 \pi T^{\alpha \beta}\right)(-g)^{1 / 2} \delta g_{\alpha \beta} \xi^{\mu} d \sigma_{\mu} .
\end{aligned}
$$

Then Eq. (6.6) reduces to the very useful form

$$
\begin{aligned}
-\oint_{\partial \mathfrak{X}} & \frac{1}{16 \pi}\left(G^{\alpha \beta}-8 \pi T^{\alpha \beta}\right)(-g)^{1 / 2} \delta g_{\alpha \beta} \xi^{\mu} d \sigma_{\mu}+\oint_{\partial \mathfrak{X}}\left(\delta \mathfrak{T}^{\mu}{ }_{\nu}-\frac{1}{2} \mathfrak{T}^{\alpha \beta} \delta g_{\alpha \beta} \delta_{\nu}^{\mu}\right) \xi^{y} d \sigma_{\mu} \\
& =\oint_{\partial \mathfrak{X}} \delta\left[\mathfrak{t}_{N}^{\mu}{ }^{\mu} \cdot \xi^{\nu}+\mathfrak{T}^{\mu}{ }_{\nu} \xi^{\nu}\right] d \sigma_{\mu}
\end{aligned}
$$

which is accordingly an identity for any $\mathfrak{X}$ whenever $g_{\mu \nu}$ and $T_{\mu \nu}$ are invariant under $\xi$.

Since we are particularly concerncd here with energy, we assume that $\xi$ is timelike at spatial infinity. We then have, for perfect fluids, the generalization of the Bardeen [2] Hartle and Sharp [14] variational principles to arbitrary configurations without any particular spatial symmetries. With suitable modifications the theorem can be made applicable to any matter fields, and we return to this point later.

TheOREM 6.4. A set $\left(g_{\alpha \beta}, \mathfrak{N}^{\alpha}, S\right)$ invariant under an asymptotically timelike killing vector $\xi$, is a solution of Einstein's equations if and only if its integral $\xi$-momentum (defined on any asymptotically regular hypersurface $\mathscr{H}$ that nowhere is parallel to $\xi$, and which has a normal field $n_{\alpha}$ ) is an extremum against all perturbations in $\mathscr{H}$ that obey the following restrictions.

(a) The number of particles is constant if $V_{\alpha} \xi^{\alpha} \neq 0$

$$
\delta \mathfrak{\cap}^{\beta} n_{\beta}=0 \quad \text { or } \quad V_{\alpha} \xi^{\alpha}=0 ;
$$

(b) the specific entropy is constant $\delta S=0$;

(c) the perturbation in $V_{\alpha}$ is restricted by

$$
P^{\beta} U^{\alpha} \delta V_{\beta}=0
$$

where

$$
P_{\alpha}^{\beta} \equiv \delta^{\beta}{ }_{\alpha}-\xi^{\beta} n_{\alpha} /\left(\xi^{\mu} n_{\mu}\right)
$$

projects vectors along $\xi$ onto $\mathscr{H}$; and 
(d) if the set $\left(g_{\alpha \beta}, \mathfrak{N}^{\alpha}, S\right)$ is invariant under motions along a vector field $\eta$ that commutes with $\xi(\underset{\xi}{\mid} \eta=0)$, then the perturbations are also so invariant. (This applies in particular, of course, to $\xi^{\alpha}$ itself.)

Before proving this theorem, we think it would be helpful to make the following remarks. First, restrictions (a)-(c) are chosen to make the integrand of the surface integral of Lemma 6.3 vanish, thereby satisfying the restriction on the perturbations that was necessary in Theorem 6.2. However, the vanishing of this integrand is only one condition at each point of $\mathscr{H}$. We have made it three in order to narrow the class of perturbations one need consider and, more importantly, to make separate restrictions on what one considers to be physically independent variables: particle number, entropy, and velocity. We thereby obtain the relativistic versions of exactly the three counterexamples mentioned in the proof of Theorem 4.1. (Note that restriction (c) above is the relativistic version of the counterexample that involved adding velocity parallel to an existing velocity. Because the tensor $P^{\beta}{ }^{\beta}$ projects vectors along $\xi^{\kappa}$ onto $\mathscr{H}$, one is forbidden by (c) from adding momentum conjugate to the three-velocity $P^{\beta}{ }_{x} U^{\alpha}$.) Our second remark is that, as will become clear in the proof, restriction (d) is only a convenience. The theorem would be true without it, but it is another useful restriction on the class of perturbations one need consider. Our final remark is that it is actually permissible for $\xi$ to be parallel to $\mathscr{H}$ on a set of measure zero in $\mathscr{H}$; the theorem then involves the Einstein equations being satisfied everywhere else, whence continuity extends it to the awkward points.

Proof of Theorem 6.4. We call the three integrals in Eq. (6.10) I, II, and III. respectively. We choose $d \mathfrak{X}$ to consist of two asymptotically regular hypersurfaces $\mathscr{H}$ and $\mathscr{H}^{\prime}$ and the cylinder at infinity joining them. We let $\delta$ be zero everywhere on $x$ except on $\mathscr{H}$, so all the integrals are now restricted to $\mathscr{H}$. This is the great simplification introduced by assuming a killing vector. One can focus on perturbations obeying constraints only on the hypersurface whose mass is being perturbed, rather than in some region $\mathfrak{X}$ bounded partly by it. Now consider perturbations obeying constraints (a)-(c) of the theorem, so that the integrand II vanishes. These are at most three constraints, which may be satisfied by choosing the five perturbations $\left(\delta \mathfrak{M}^{x}, \delta S\right)$ in such a way as to leave $\delta g_{\alpha \beta}$ arbitrary ${ }^{22}$ Since integral III is simply the perturbation in $P[\xi, \mathscr{H}]$, it is clear that $P[\xi, \mathscr{H}]$ is an extremum if and only if integral I vanishes for all $\delta g_{\alpha \beta}$, which will be true if and only if the unperturbed systems satisfy the Einstein equations. This proves the theorem when the perturbations obey only restrictions (a)-(c).

In order to show that the further restriction (d) may be imposed, we need only show that adding it does not affect the proof that the extremality of $P[\xi, \mathscr{H}]$ implies the

22 This involves a mild generality assumption. In fact one can show that there exist situations where it is not possible to fulfill these three constraints while leaving $\delta g_{\alpha \beta}$ arbitrary everywhere, but in these cases the restriction on $\delta g_{\alpha \beta}$ will probably never occur except on a set of points of $\mathscr{H}$ of measure zero. In any case one can always combine restrictions (a) and (c) into a single one at these points, thereby choosing $\delta N^{\alpha}$ and $\delta S$ in such a way as to leave $\delta g_{\alpha \beta}$ arbitrary and still make the integrand of II vanish. 
Einstein equations. In the particular case where the invariance $\eta$ is a motion off $\mathscr{H}$ (as it is for $\xi$ ), the proof is obviously unaffected, since the values of $\underset{n}{£} \delta g_{\alpha \beta}, \underset{n}{£} \delta \mathfrak{N}^{4}$, and $£ \delta S$ never enter integrals I and II of Eq. (6.10). On the other hand, if the integral curves of $\eta$ lie in $\mathscr{H}$, or at least in some region $U$ of $\mathscr{H}$, it is possible to construct coordinates $(x, y, \lambda)$ in $U$ such that curves of constant $x$ and $y$ are integral curves of $\eta$. By hypothesis $\xi^{\alpha}$ and $\left(G^{\alpha \beta}-8 \pi T^{\alpha \beta}\right)(-g)^{1 / 2}$ are independent of $\lambda$, and $d \sigma_{\mu} \rightarrow d x d y d \lambda$, so integral I of Eq. (6.10) depends only on the integral of $\delta g_{\alpha \beta}$ along $\lambda$ through $U$. One loses no generality, then, in taking $\delta g_{\alpha \beta}$ to be a constant in $\lambda$ in that region. Similarly, integral II can still be made to vanish as before. Therefore the class of allowed perturbations can be required to be invariant under $\eta$ without loss of generality.

We will show that the Bardeen [2] variational principle is a special case of this in the next section. First we give the appropriate Newtonian versions of the important results. Equation $(6.3 \mathrm{~N})$ can be written, by discarding spatial divergences, as

$$
\begin{aligned}
& \int_{t_{\mathbf{1}}}^{t_{2}} d t \int d^{3} x\left[\left(-(1 / 4 \pi) \nabla^{2} \Phi+\rho_{0}\right) \partial_{t} \Phi+n T \partial_{t} S\right. \\
& \left.\quad+\left(\Phi+\frac{1}{2} V^{2}+h\right) \partial_{t} \rho_{0}+\rho_{0} \mathbf{V} \cdot \partial_{t} \mathbf{V}\right]=\left[\mathscr{E}_{t_{1}}^{t_{2}},\right.
\end{aligned}
$$

where $\mathscr{E}$ is the total energy integral.

In discussing perturbations of this it is most natural to use the isometric map $g$ between the perturbed and unperturbed three-spaces, which is unique up to rigid translations and rotations. This is normally called the Eulerian perturbation, and we shall call its derivation $\delta_{g}$. Perturbing the above equation when $\Phi, S, \rho_{0}$, and $\mathbf{V}$ are time independent gives

$$
\begin{aligned}
& {\left[\int d ^ { 3 } x \left\{\left(-(1 / 4 \pi) \nabla^{2} \Phi+\rho_{0}\right) \delta_{g} \Phi+n T \delta_{m} S\right.\right.} \\
& \left.\left.\quad+\left(\Phi+\frac{1}{2} V^{2}+h\right) \delta_{g} \rho_{0}+\rho_{0} V^{i} \cdot \delta_{g} V_{i}\right\}-\delta \mathscr{E}\right]_{t_{1}}^{t_{2}}=0 .
\end{aligned}
$$

Since the perturbations at $t_{1}$ and $t_{2}$ are independent, this equation gives $\delta \mathscr{E}$ at any time. (No subscript is placed on $\delta \mathscr{E}$ because it is an integral, independent of the mapping.) A completely general mapping $q$ between the perturbed and unperturbed manifold is generated from $g$ by Eq. (6.5)

$$
\delta_{q}=\delta_{g}-\underset{\mathfrak{n}}{\mathfrak{k}}
$$

where $\eta$ represents some displacement. The perturbations of the basic variables are found from

$$
\delta_{q} S=\delta_{g} S-\eta \cdot \nabla S
$$

with a similar equation for $\Phi$,

$$
\delta_{q} V_{i}=\delta_{g} V_{i}-\eta^{j} \nabla_{j} V_{i}-V_{j} \nabla_{i} \eta^{j}
$$


and for the density of particles

$$
\delta_{q}\left(\rho_{0} g^{1 / 2}\right)=g^{1 / 2}\left[\delta_{g} \rho_{0}+\nabla \cdot\left(\rho_{0} \eta\right)\right],
$$

where $g^{1 ; 2}$, the square root of the determinant of the metric, is the Jacobian of the transformation from Cartesian coordinates to whatever general coordinates are being used. Then we can deduce that the change in the total energy, $\delta \mathscr{E}==\delta_{g} \mathscr{E}==\delta_{\imath} \mathscr{E}$ is

$$
\begin{aligned}
& \delta \ell^{\prime}=\int d^{3} x\left\{\left(-(1 / 4 \pi) \nabla^{2} \Phi+\rho_{0}\right) \delta_{q} \Phi+n T \delta_{q} S+\rho_{0} V^{i} \cdot \delta_{r_{q}} V_{i}\right. \\
& \cdots\left(\Phi+\stackrel{1}{2} V^{2}-h\right) g^{-(1 / 2)} \delta_{q}\left(\rho_{0} g^{1 / 2}\right)-\rho_{0} \eta \cdot\left[(\mathbf{V} \cdot \nabla) \mathbf{V} \div\left(1 / \rho_{0}\right) \nabla p-\nabla \Phi\right]_{i} .
\end{aligned}
$$

This is to be regarded as the analog of Eq. (6.10). It immediately gives [cf. a more restricted version in Lynden-Bell [22]]

THEOREM 6.40. A time-independent set $\left(\Phi, \rho_{0}, S, \mathrm{~V}\right)$ is a solution of Newton's gravitational field equation and Euler's equation if and only if its total energy is an extremum against all stationary perturbations that obey the following restrictions.

(a) The number of particles is constant $\delta_{\eta}\left(\rho_{0} g^{1 / 2}\right)==0$;

(b) the specific entropy is constant $\delta_{q} S=0$;

(c) the velocity perturbation is perpendicular to any existing velocity $V^{i} \cdot \delta_{\|} V_{i} \ldots 0$; and

(d) if the set $\left(\Phi, \rho_{0}, S, \mathrm{~V}\right)$ is invariant under motions along a spatial vector field $\zeta$ then the perturbations are also so invariant.

\section{(e) Relation to Bardeen Variational Principle}

To make explicit the relation of Theorem 6.4 to Bardeen's result [2], we consider the configurations he dealt with: sets $\left(g_{\alpha \beta}, \mathfrak{I} \mathfrak{H}^{\alpha}, S\right)$ invariant under the asymptotically timelike killing vector $\hat{\partial} / \hat{c} t$ and a commuting axial killing vector $\partial / \partial \phi$, and which are invariant under the reflection $(t, \phi) \rightarrow(-t,-\phi)$. [Bardeen does not actually mention this reflection symmetry, but he does use it. It excludes meridional (convective) circulation. See Carter [47].] Only perturbations that are also reflection-symmetric will contribute to first-order changes in the mass. Remembering that $V_{\phi}$ is usually called $j$, the angular momentum per particle, we have proved the following corollary of Theorem 6.4 .

COROllary 6.5. (Bardeen [2]). An axisymmetric, stationary set $\left(\mathrm{g}_{\alpha \beta}, \mathfrak{N}^{\circ}, S\right)$ invariant under $(t, \phi)$ reflection satisfies Einstein's equations if and only if its integral mass is an extremum against all axisymmetric, momentarily stationary, reflectionintariant perturbations that preserve particle number, entropy, and specific angular momentum.

What does it mean that the perturbation be axisymmetric and momentarily stationary? Axisymmetry means that the function $\delta g_{\alpha \beta}$ on the unperturbed manifold $M$ be independent of $\phi$. This means that the metric $g_{\alpha \beta}^{\prime}$ of $M^{\prime}$ must also be axisymmetric. with a Killing vector $\partial / \partial \phi^{\prime}$ which is mapped by $e$ into $\partial / \partial \phi$. Similarly, stationarity im- 
plies the existence on $M^{\prime}$ of a Killing vector $\partial / \partial t^{\prime}$ which is mapped into $\partial / \partial t$. Thus, given an acceptable map $e$, another one $e^{\prime}$ can be obtained by translation along a vector in $M$ orthogonal to $\partial / \partial t$ and $\partial / \partial \phi$ (cf. Eq. (6.5)). In Bardeen's language, this is the freedom to move "rings" of the fluid around. These maps are not Lagrangian maps, which carry integral curves of the fluid's four-velocity into one another, as the following argument shows. The four velocity $\mathbf{U}$ is a linear combination of $\partial / \partial t$ and $\partial / \partial \phi$, so we need only consider the map of the subspace $\left(\partial / \partial t^{\prime}, \partial / \partial \phi^{\prime}\right)$ into $(\partial / \partial t, \partial / \partial \phi)$. This map is completely determined by the requirement that it map certain integral curves of $t^{\prime}$ and $\phi^{\prime}$ into those of $t$ and $\phi$. It will generally not map the integral curves of another vector field $\mathbf{U}^{\prime}$ on $M^{\prime}$ into those of any given $\mathbf{U}$ on $M$, unless $\mathbf{U}^{\prime}$ is proportional to the same linear combination of $\partial / \partial t^{\prime}$ and $\partial / \partial \phi^{\prime}$ as that which $\mathbf{U}$ is of $\partial / \partial t$ and $\partial / \partial \phi$. This happens only if the angular velocity is not perturbed. But the angular velocity will inevitably be perturbed in order to keep the angular momentum the same, so an acceptable map $e$ in Corollary 6.5 cannot be Lagrangian.

Notice that in the present case, specification of the metric on $M^{\prime}$ and the map $e$ completely determines the perturbation of all the fluid variables. This is because the reflection symmetry allows only $\delta \mathfrak{N}^{t}$ and $\delta \mathfrak{R}^{\phi}$ and $\delta S$ to be nonzero, while the three constraints of Theorem 6.4 determine $\delta \mathfrak{N}^{t}(=0)$, $\delta \mathfrak{N}^{\phi}$, and $\delta S(=0)$ in terms of $\delta g_{\alpha \beta}$, once $e$ is given. From this point of view, $\delta g_{\alpha \beta}$ is the only free variable. Bardeen identifies free variables differently in order to make a formal analogy with the Ncwtonian treatment. First he establishes an "Eulerian" map $g$ which preserves a certain gauge condition on the metric, and which is different from the maps we consider above, calling $\delta_{g} g_{\alpha \beta}$ the "metric perturbation." He then obtains one of our maps $e$ related to $g$ by a vector field, whose two components are orthogonal to $\partial / \partial t$ and $\partial / \partial \phi$ and provide the two remaining degrees of freedom. These variables would have to be supplemented in order to apply the variational principle to axisymmetric stationary systems that lack the reflection symmetry, where there are more free perturbations than just $\delta_{e} g_{\alpha \beta}$.

\section{(f) General Remarks}

Theorem 6.4 provides a variational approach to the construction of stationary stellar models that lack the symmetries that have usually been assumed, in particular nonaxisymmetric solutions like the Dedekind ellipsoids and their relativistic counterparts, if they exist. It may, in particular, give an efficient method for getting approximate solutions, which may then be refined by the self-consistent field methods used lately on axisymmetric models (e.g. Ostriker and Bodenheimer [26], Butterworth and Ipser [6]). In using this method one loses no generality by restricting oneself to perturbations that solve the initial-value equations, since an extremal solution does in fact satisfy them. But such a restriction may not be calculationally efficient, and is not necessary for the method to work. ${ }^{23}$

${ }^{23}$ As Friedman and Schutz [12] have shown, a stable star in general relativity is a minimum of the energy among all nearby momentarity stationary configurations that do satisfy the initial-value constraints. By allowing configurations that do not satisfy the constraints, one loses minimality but retains extremality of the integral mass we have defined. 
Theorem 6.4 also gives interesting insight into the ways in which the mass of a stationary solution can be changed to first order, and the effect of such changes.

COROLlaRY 6.6. The integral mass of a stationary solution can be changed to first order only by changing the number of particles $N$, the specific entropy $S$, or the specific momentum $V$ conjugate to the existing velocity. The resulting changes in the mass are ${ }^{24}$

(a) addition of a single particle at constant $S$ and $V_{\alpha}: \delta M=-\mu U_{\alpha} \xi^{\alpha}$. [Newtonian: $\delta E=\left(\Phi+\frac{1}{2} V^{2}+h\right) m$, where $m$ is the mass of the particle. $]$

(b) Addition of specific entropy $\delta S$ to a fluid element containing $N$ particles, at constant $N$ and $V_{\alpha}$ (i.e., adding total entropy $\left.N \delta S\right): \delta M=T N \delta S\left(\xi^{\alpha} n_{\alpha}\right) /\left(U^{\alpha} n_{\alpha}\right)$ where $n_{\alpha}$ is normal to the hypersurface. [Newtonian: $\delta E=N T \delta S$.]

(c) Addition of specific momentum $\delta V_{\alpha}$ to a fluid element containing $N$ particles, at constant $N$ and $S$ (i.e., adding total momentum $\left.N \delta V_{\alpha}\right): \delta M=N\left(P_{\beta}^{\alpha} U^{\beta} \delta V_{\alpha}\right)\left(\xi^{\mu} n_{\mu}\right)$ $\left(U^{v} n_{v}\right)$. When $n_{\alpha}=(1,0,0,0)$ and $\xi^{\alpha}=(1,0,0,0)$ this is $\delta M=N U^{i} \delta V_{i} / U^{0}$. [Newtonian: $\delta E=m N \mathbf{V} \cdot \delta \mathbf{V}$.]

Proof. These expressions follow in a straightforward manner from expressions (6.6), (6.9), and Theorem 6.4 [in the Newtonian case, from (6.10N)]. One needs to use the fact that the number of particles in a fluid element is $d N==\mathfrak{N}^{\alpha} d \sigma_{\alpha}$ (Newtonian: $\left.d N=\rho_{0} d^{3} x / m\right)$.

A few comments are in order. From (a) one can call $-\mu U_{\alpha} \xi^{\alpha}$ the injection energy at constant specific entropy and specific momentum. Thorne [44], discussing convection in rotating stars, defined the injection energy to be $\mu\left(\xi^{\alpha} n_{\alpha}\right) /\left(U^{\alpha} n_{\alpha}\right)$. The difference between our definition and his is that his injection process holds the total momentum of the star constant, rather than the specific momentum of the fluid element. (One may expect that Thorne's definition is better suited than ours to a discussion of convection on stars.) It is easy to derive his injection energy from ours, since the specific momentum of an element containing $N$ particles must change by $\delta V_{\alpha}=-V_{\alpha} / N$ upon addition of a single particle at constant total momentum. Then combining (a) and (c) gives $\delta M=-\left(U^{v} V_{v}\right)\left(\xi^{\alpha} n_{\alpha}\right) /\left(U^{\beta} n_{\beta}\right)$, which is the same as Thorne's when the definition of $V_{\mu}$ is used. [The Newtonian analog of Thorne's injection energy is $m\left(\Phi-\frac{1}{2} V^{2}+h\right)$.]

This corollary gives the changes in the integral mass $P[\xi, \mathscr{H}]$. This is the change in the total mass when the region $\mathscr{H}$ over which the integral is taken has only one boundary, at spatial infinity, as we have assumed throughout this paper. But if it had an inner boundary (for instance at the horizon of a black hole) then Corollary 6.6 would give the change in the difference between the surface integrals on the boundaries (cf. Eq. (3.10)). If the inner boundary were a black-hole horizon, then the change in the surface integral on it should give the change in the black-hole's $\xi$-momentum (though we have not yet explicitly verified this). Then one would recover the mass-

${ }^{24}$ In comparing the Newtonian and relativistic expressions one must bear in mind that we have found it convenient to let $\mu$ be the relativistic enthalpy per particle (having dimensions of energy) while $h$ is nonrelativistic enthalpy per unit mass (having dimensions of velocity squared). This accounts for the extra factor of $m$ in the Newtonian expressions for (a) and (c). 
formula of Bardeen, Carter and Hawking [3]. Note, however, that the BardeenCarter-Hawking result was proved only for stationary perturbations, while Corollary 6.6 applies to any perturbation.

The extension of Theorem 6.4 to general matter fields relies on part (a) of Lemma 6.3. As long as this constraint permits free variations of the $g_{\alpha \beta}$ the theorem will still be valid. But, as remarked in connection with Theorem 6.2 , one will conclude from extremality of the $\xi$-momentum only that the Einstein equations are satisfied; for general systems this does not necessarily imply the matter field equations. A partial illustration of this is, in fact, provided by the Newtonian version, Eq. $(6.10 \mathrm{~N})$ : Newton's field equation does not imply Euler's equations. We still get Euler's equations from the variational principle because we demand extremality of the energy against all translations of the fluid elements as well as all perturbations in place. (Compare Eq. $(6.10 \mathrm{~N})$ with the version before it involving only $\delta_{g}$. .) In general relativity this distinction between "translations" and "in place" perturbations is not well defined, so the relativistic version automatically incorporates these general variations as a result of its coordinate-invariance.

\section{APPENDIX: Perfect-Fluid Potentials}

According to Theorem 4.1, it is impossible to construct an unconstrained variational principle for the perfect fluid in the Eulerian variables. Therefore one is forced either to constrain the variations or to introduce non-Eulerian variables. The approach using constraints was dealt with thoroughly in Section 4 . We consider that using constraints keeps the principle closest to the underlying physics, which is a great advantage in Section 6. But the specialist may wish to look at the alternative approach of introducing potentials, which has in the past decade led to some interesting formalisms for dealing with the equations of hydrodynamics.

We can keep the variations in $\mathfrak{N}^{\alpha}$ and $S$ completely free by introducing Lagrange multipliers for the constraints. For simplicity we will assume that we are interested only in perfect fluids that obey conservation of particle number, and hence of entropy. There are two constraints. The entropy constraint may be expressed as

$$
\mathfrak{N}^{\alpha} \partial_{\alpha} S=0 .
$$

The other constraint is the preservation of particle identity, as discussed in Section 4. The way to introduce this was first suggested by Lin [39]. Briefly, one defines three functions $X, Y$, and $Z$, which are considered to be particle labels. These labels are then preserved by the motion of the fluid, so that the boundary conditions $\delta X=\delta Y=$ $\delta Z=0$ on $\partial \mathfrak{X}$ prevent one from varying the endpoints of the world lines

$$
\mathfrak{R}^{\alpha} \partial_{\alpha} X=\mathfrak{N}^{\alpha} \partial_{\alpha} Y=\mathfrak{N}^{\alpha} \partial_{\alpha} Z=0 .
$$

Using a Lagrange multiplier for each constraint we obtain the unconstrained Lagrangian

$$
\mathfrak{L}^{\prime}=\rho(-g)^{1 / 2}+\theta \mathfrak{N}^{\alpha} \partial_{\alpha} S+A \mathfrak{N}^{\alpha} \partial_{\alpha} X+B \mathfrak{N}^{\alpha} \partial_{\alpha} Y+C \mathfrak{N}^{\alpha} \partial_{\alpha} Z
$$


This cumbersome expression can be simplified somewhat by making use of Pfaff's theorem (cf. Schutz [34]). One can see that by varying $A, X$, etc. independently in (A3) we will find them all conserved along $U^{\alpha}$. They are therefore only functions of three "spatial" variables. In particular, one can therefore express $A, B$, and $C$ as functions of $X, Y$, and $Z$. For such a set of functions, Pfaff's theorem says there always exist three other functions $\alpha(X, Y, Z), \beta(X, Y, Z)$, and $\phi(X, Y, Z)$ such that

$$
A(X, Y, Z) d X+B(X, Y, Z) d Y+C(X, Y, Z) d Z=d \phi \ldots x d \beta .
$$

This transformation seems first to have been used by Clebsch [9]. It enables Lin's constraint to be simplified in the Lagrangian to

$$
\mathfrak{Z}^{\prime}=\rho(-g)^{1 / 2}+\theta \mathfrak{N}^{\alpha} \partial_{\alpha} S+\mathfrak{N}^{\alpha} \partial_{\alpha} \phi+\alpha \mathfrak{N}^{\alpha} \partial_{\alpha} \beta
$$

Holding the metric fixed, we use Eqs. (4.13) and (4.15) to obtain the Euler-Lagrange equations

$$
\begin{aligned}
& \delta \mathfrak{N}^{\alpha}:-\mu U_{\alpha}+\partial_{\alpha} \phi+\theta c_{\alpha} S+\alpha \hat{\sigma}_{\alpha} \beta=0 \\
& \delta S: n(-g)^{1 / 2} T-\partial_{\alpha}\left(\theta \mathfrak{M}^{\alpha}\right)=0 \\
& \delta \theta: \mathfrak{N}^{\alpha} \partial_{\alpha} S=0 \\
& \delta \phi: \partial_{\alpha} \mathfrak{N}^{\alpha}=0 \\
& \delta \alpha: \mathfrak{N}^{\alpha} \partial_{\alpha} \beta=0 \\
& \delta \beta: \partial_{\alpha}\left(\alpha \mathfrak{N}^{\alpha}\right)=0
\end{aligned}
$$

These are identical to the "velocity-potential equations" derived by Schutz [34], and equivalent to those of Schmid [32]. They were first derived in Newtonian form by Scliger and Whitham [37]. They are completely equivalent to Eqs. (4.1) (cf. Schutz [34]).

How do Eqs. (A6) embody the constraints? The entropy contraint comes out explicitly in (A6c), but the Lin constraints come out apparently only on $\alpha$ and $\beta$ in (A6e) and (A6f). However, conservation of particles comes out explicitly in (A6d) even though it was not put in explicitly. This is effectively the third Lin constraint, since conservation of particles is implied by preservation of the identity of particles.

Pfaff's transformation has, therefore, put the constraints in a slightly more convenient (or at least economical) form. We could have taken the multiplier form of the constraints to be

$$
-\phi \hat{\sigma}_{\alpha} \mathfrak{l}^{\alpha}+\alpha \mathfrak{N}{ }^{\alpha} \hat{c}_{\alpha} \beta
$$

which differs from their form in (A5) by a divergence. In this form it appears as if conservation of particles and only one of Lin's constraints is needed. This has given rise to remarks in Eckart [10] and Serrin [39] that mysteriously only one Lin constraint seems to be needed, not the three in (A2). But this remark is erroneous, because it forgets that conservation of particles, which follows from Lin's constraints, is also needed in (A7). The proper point of view is that all three of Lin's constraints are in (A7), but expressed economically via Pfaff's theorem. 
Equations (A6) and the normalization of $U^{\alpha}$,

$$
U^{\alpha} U_{\alpha}=-1
$$

provide an equation for $\phi$

$$
U^{\alpha} \partial_{\alpha} \phi=-\mu .
$$

It is perhaps interesting that the value of the Lagrangian $\mathfrak{L}^{\prime}$ in (A5) is, via (A6) and (A8),

$$
\mathfrak{L}^{\prime}=\rho(-g)^{1 / 2}-n \mu(-g)^{1 / 2}=-p(-g)^{1 / 2} .
$$

Although the value of $\mathfrak{Q}^{\prime}$ is irrelevant to its extremal properties, this equation suggests that the pressure could also serve as the Lagrangian. This is, in fact, the case: Seliger and Whitham [37], Schmid [32], and Schutz [34] all use $p$ as their Lagrangians. In these approaches, Eq. (A6a) is assumed as the general form of $U^{\alpha}$ (cf. Schutz [34] for proof of its generality) and all the other equations are derived by varying $p(-g)^{1 / 2}$ with respect to $\phi, \alpha, \beta, \theta$, and $S$. The flux $\mathfrak{N}^{\alpha}$ is not even a variable, since its EulerLagrange equation is assumed a priori; these approaches are, then, the purely potential alternative to the constrained version in Section 4.

The potential $\theta$, incidentally, has a long history, which has been traced by Schmid [32]. Van Dantzig [46] called it the "thermasy" and the naffle has stuck. In the interesting canonical approach of Grosjean [13], the thermasy is used as a canonical time, whose conjugate momentum, the effective Hamiltonian, is the entropy. This conjugate relationship between the pairs $(\theta, S)$ and $(\alpha, \beta)$ is a striking feature of the potential version of hydrodynamics. In the case of the rotating star $[35,36]$, the potential $\beta$ is the comoving angular coordinate $\phi-\Omega t$, while $\alpha$ is the momentum conjugate to it: the angular momentum per particle in the fluid.

\section{REFERENCES}

1. J. L. Anderson and P. G. Bergman, Phys. Rev. 83 (1951), 1018.

2. J. M. BARDEEN, Astrophys. J. 162 (1970), 71.

3. J. M. Bardeen, B. Carter, and S. W. Hawking, Comm. Math. Phys. 31 (1973), 161.

4. F. J. Belinfante, Physica 7 (1940), 449.

5. D. R. Brill and S. Deser, Phys. Rev. Lett. 20 (1968), 75.

6. E. M. Butterworth AND J. R. IPSER, Astrophys. J. Lett. 200 (1975), L103.

7. M. Cantor, A. Fischer, J. Marsden, N. \& Murchadha, and J. York, Comm. Math. Phys. 49 (1976), 187.

8. B. CARTER, in "Les Astre Occlus," Proceedings of 1972 Les Houches Summer School (C. DeWitt and B. S. DeWitt, Eds.), Gordon \& Breach, New York, 1973.

9. A. CleBsCh, J. Reine Angew. Math. 56 (1859), 1.

10. C. ECKART, Phys. Fluids 3 (1960), 421.

11. E. FERMI, "Thermodynamics," Dover, New York, 1936.

12. J. L. Friedman and B. F. Schutz, Astrophys. J. 200 (1975), 204.

13. P. V. Grosjean, Bull. Soc. R. Sci. Liege 41 (1972), 31.

14. J. B. Hartle AND D. H. Sharp, Astrophys. J. 147 (1967), 317.

15. S. W. HAWking AND G. F. R. Ellis, "The Large Scale Structure of Space-Time," Cambridge Univ. Press, London/New York, 1973. 
16. A. Komar, Phys. Rev. 113 (1958), 934.

17. R. M. Kulsrud, Astrophys. J. 152 (1968), 1121.

18. L. Landau and E. M. Lifshitz, "The Classical Theory of Fields," 2nd ed., Pergamon, New York, 1962.

19. F. London, "Superfluids Vol. II," Dover, New York, 1954.

20. A. Lichnerowicz, C. Rendus 222 (1946), 432.

21. A. Lichnerowicz, "Theories Relativistes de la Gravitation et de l'Electromagnetisme," Paris: Masson, 1955.

22. D. Lynden-Bell, Ph.D. Thesis, Univ. of Cambridge (Clare College), 1960.

23. D. Lynden-Bell and J. P. Ostriker, Mon. Not. Roy. Astron. Soc. 136 (1967), 293.

24. C. W. Misner, K. S. Thorne, and J. A. Wheeler, "Gravitation," Freeman, San Francisco, 1973.

25. N. O Murchadha, Phys. Lett. 60 A (1977), 177.

26. J. P. OStriker AND P. BODenheimer, Astrophys. J. 151 (1968), 1089.

27. W. PAul. "Theory of Relativity," Pergamon, New York, 1958.

28. P. Penfield, Phys. Fluids 9 (1966), 1184.

29. L. Rosenfeld, Acad. Roy. Belg. Cl. Sci. 18 (1938), 3.

30. M. Ruderman, Ann. Rev. Astron. Astrophys. 10 (1972), 427.

31. L. A. Schmid, in "A Critical Review of Thermodynamics" (B. Stuart, B. Gal-Or, and A. J. Brainard, Eds.), pp. 161-202, Mono, Baltimore, 1970.

32. L. A. Schmid, Pure Appl. Chem. 22 (1970), 493.

33. L. A. SCHMID, 1972 Report X-641-72-467 from NASA, Goddard Space Flight Center, Greenbelt, Maryland.

34. B. F. Schutz, Phys. Rev. D 2 (1970), 2762.

35. B. F. Schutz, Astrophys. J. Suppl. 24 (1972), 319 (No. 208).

36. B. F. Schutz, Astrophys. J. Suppl. 24 (1972), 343 (No. 208).

37. R. L. Seliger and G. B. Whitham, Proc. Roy. Soc. London A 305 (1968), 1.

38. R. Serini, R. C. Accad Lincei (5) 27 (1918), (first part), 235.

39. J. Serrin, in "Handbuch der Physik," Vol. 8, Springer-Verlag, Berlin, 1959.

40. R. Sorkin, Gen. Rel. and Grav, in press.

41. A. H. Taub, in "Proceedings of the First Symposium of Applied Mathematics," pp. 148-157, American Mathematical Society, New York, 1949.

42. A. H. TAUB, Phys. Rev. 94 (1954), 1468.

43. K. S. ThORNe, in "High Energy Astrophysics" (C. De Witt, E. Schatzman, P. Veron, Eds.), Vol. 3, Gordon \& Breach, New York, 1967.

44. K. S. Thorne, in "General Relativity and Cosmology," Proceedings of Enrico Fermi Summer School Course 47 (R. K. Sachs, Ed.), Academic Press, New York, 1971.

45. A. Trautman, in "Gravitation: an Introduction to Current Research" (L. Witten, Ed.), Wiley, New York, 1962.

46. D. Van Dantzig, Proc. Kon. Akad. Wetenschappen Amst. 42 (1939), 601.

47. B. CARTer, J. Math. Phys. 10 (1969), 70. 